\title{
QCD in the color-flow representation
}

\author{
W. Kilian, ${ }^{a}$ T. Ohl, ${ }^{b}$ J. Reuter ${ }^{c}$ and C. Speckner ${ }^{d}$ \\ ${ }^{a}$ Universität Siegen, Department Physik, \\ Walter-Flex-Str. 3, 57068 Siegen, Germany \\ ${ }^{b}$ Universität Würzburg, Institut für Theoretische Physik und Astrophysik, \\ Emil-Hilb-Weg 22, 97074 Würzburg, Germany \\ ${ }^{c}$ DESY Theory Group, \\ Notkestr. 85, 22603 Hamburg, Germany \\ ${ }^{d}$ Universität Freiburg, Physikalisches Institut, \\ Hermann-Herder-Str. 3, 79104 Freiburg, Germany \\ E-mail: kilian@physik.uni-siegen.de, ohl@physik.uni-wuerzburg.de, \\ juergen.reuter@desy.de, christian.speckner@physik.uni-freiburg.de
}

AbstRACT: For many practical purposes, it is convenient to formulate unbroken nonabelian gauge theories like QCD in a color-flow basis. We present a new derivation of $\mathrm{SU}(N)$ interactions in the color-flow basis by extending the gauge group to $\mathrm{U}(N) \times \mathrm{U}(1)^{\prime}$ in such a way that the two $\mathrm{U}(1)$ factors cancel each other. We use the quantum action principles to show the equivalence to the usual basis to all orders in perturbation theory. We extend the known Feynman rules to exotic color representations (e.g. sextets) and discuss practical applications as they occur in automatic computation programs.

KeYwords: QCD, Scattering Amplitudes, 1/N Expansion

ARXiv EPRINT: 1206.3700 


\section{Contents}

1 Introduction 2

2 The model 4

2.1 QCD preliminaries 4

2.2 The color-flow representation 5

$\begin{array}{lll}2.3 & \text { Singlet and phantom gluons } & 6\end{array}$

$\begin{array}{lll}2.4 & \text { Color-flow QCD } & 7\end{array}$

2.5 Example 8

3 Field-theoretical considerations $\quad 8$

$\begin{array}{llr}3.1 & \text { Algebraic identities at lowest order } & 9\end{array}$

$\begin{array}{lll}3.2 & \text { Fock-space cancellation } & 10\end{array}$

$\begin{array}{lll}3.3 & \text { Symmetries } & 11\end{array}$

4 Renormalization $\quad 11$

$\begin{array}{ll}\text { 4.1 Conditions imposed on the effective action } & 12\end{array}$

4.2 Lowest-order solution 13

$\begin{array}{lll}4.3 & \text { Inductive renormalization } & 13\end{array}$

5 Applications: tree-level amplitudes $\quad 15$

$\begin{array}{lll}5.1 \text { Algorithm } & 15\end{array}$

$\begin{array}{lll}5.2 & \text { Quark-antiquark scattering } & 17\end{array}$

$\begin{array}{lll}5.3 & \text { Four-jet production in } e^{+} e^{-} & 18\end{array}$

6 Applications: effective interactions from loops 19

$\begin{array}{ll}6.1 & g g \rightarrow H\end{array}$

$\begin{array}{ll}6.2 \mathrm{gg} \rightarrow \mathrm{HH} & 21\end{array}$

$\begin{array}{ll}6.3 H \rightarrow g g g & 24\end{array}$

$\begin{array}{lll}7 & \text { Conclusions } & 25\end{array}$

$\begin{array}{ll}\text { A Feynman rules for color-flow QCD } & 26\end{array}$

$\begin{array}{ll}\text { B Color-sextet particles } & 28\end{array}$ 


\section{Introduction}

The analysis of particle physics experiments at colliders depends on reliable theoretical predictions for cross sections of scattering processes. In the LHC era, hard processes with more external partons than ever have become accessible, and their analysis is essential for unveiling the physics at the Terascale. This results in a two-sided challenge: on one hand, complex Standard Model processes must be computed with unprecedented precision and on the other hand, the parameter space for many models for physics beyond the Standard Model must be scanned with sufficient accuracy. This situation has prompted the development of computer programs that can automatically compute differential cross sections and sample them efficiently on phase space starting from the specification of a Lagrangian or, equivalently, a set of Feynman rules.

In QCD, quarks and antiquarks come in three colors and gluons in eight. Thus, there are many amplitudes with different color quantum numbers that must be computed and summed in quadrature, eventually. In Feynman diagram based calculations, the contribution of each diagram factorizes into the dependence on color and the dependence on flavor, polarization and momenta. This allows to compute the color factors once and for all. However, for processes with many external particles, the number of Feynman diagrams grows factorially, and more efficient, e.g. recursive, algorithms must be used that can take advantage of the cancellations among diagrams. Unfortunately, the color dependence does not factorize for the whole scattering amplitude and new expansions, like color ordered amplitudes must be employed to allow a separate computation of color factors.

It is therefore worthwhile to investigate efficient representations of the color dependence that work for complete amplitudes, i.e. sums of Feynman diagrams. It turns out that expressing everything in reducible tensor products of the fundamental representation and its conjugate instead of higher irreducible representations is beneficial both for computation and for interfacing to parton showers, fragmentation and hadronization. If there are no interaction vertices with exotic color structures, the resulting amplitudes can always be decomposed into weighted sums of products of Kronecker- $\delta$ s, so called color flows. ${ }^{1}$

The adjoint representation of $\mathrm{U}(N)$ is isomorphic to the tensor product $N \times \bar{N}$ of the fundamental representation and its conjugate. Thus, the color coordinate $a$ of a $\mathrm{U}(N)$ gauge boson in the adjoint representation can be represented equivalently by a pair of $(i, j)$ using the decomposition $A_{i}^{j}=A^{a}\left[T^{a}\right]_{i}^{j}$. This equivalence is employed in 't Hooft's double line notation [1], in which each $\mathrm{U}(N)$-gauge boson line is replaced by a pair of matter and anti-matter lines for the purpose of computing color factors:

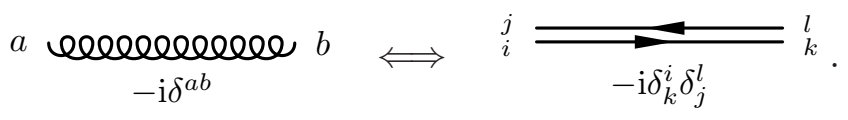

It was soon realized that the diagrammatic rules could be extended to accomodate the tracelessness of the generators of $\mathrm{SU}(N)$ [2]. Indeed, an efficient diagrammatical algo-

\footnotetext{
${ }^{1}$ If there are couplings like a totally antisymmetric vertex $\sum_{i, j, k=1}^{3} \epsilon_{i j k} \phi_{i} \phi_{j}^{\prime} \phi_{k}^{\prime \prime}$ in SU(3), the corresponding invariant tensors can also appear in the final result.
} 
rithm for computing arbitrary color factors in any gauge group was constructed [3] using a generalization of the double line notation to higher representations. ${ }^{2}$

However, the color-flow representation is more than a useful calculational device: Feynman diagrams in the double line notation bear a striking resemblance to dual diagrams $[6,7]$ and indeed the $N \rightarrow \infty$-limit is related to a theory of open strings [1]. It is therefore no accident that the successful semiclassical models for the fragmentation of quarks and gluons that are based on string dynamics [8] require a specification of the parton cross sections in the color-flow basis [9]. The earliest examples of Monte-Carlo event generators that combined the partonic cross sections for the hard interactions with QCD-inspired fragmentation models [10] provided a template for the interfacing of hard interactions with fragmentation. Similarly, a color-flow representation is used in leading order in $1 / N$ for the implementation of color coherence in parton showers [11, 12]. These interfaces became the standard HEPEVT common block [13], which includes color flow information. Originally developed for interfacing Monte-Carlo event generators for hard scattering matrix elements with libraries or external programs for parton showers, fragmentation and hadronization at LEP1, it was widely adopted and has grown into the Les Houches Accords (LHA) [16, 17].

Therefore the color-flow basis is particularly suited for the computation of partonic scattering amplitudes that will be interfaced with the other components of a Monte-Carlo event generator. Indeed, most generators for hard multi-parton scattering can provide the corresponding information. One can, however, go one step further and not only represent the final result in a color-flow basis, but use Feynman rules in a color-flow basis already for the computation. A lucent derivation of these Feynman rules was given in [18]. Since the derivation in [18] is based on the QCD Lagragian, it is not necessary to use Feynman rules at all, but one can implement modern recursive algorithms [19-21] directly in the color-flow basis [22-24].

However, the derivation [18] of the color-flow representation is incomplete in two directions: firstly, there is no consideration of interactions with more exotic color structures, in particular beyond QCD with fermionic matter in the fundamental representation, and secondly the discussion is deliberately confined to tree level amplitudes. These two limitations are in fact related, and overcoming them is not only of theoretical interest: the most important light Higgs production channel at LHC involves the dimension-5 operator $H \operatorname{Tr}\left(F_{\mu \nu} F^{\mu \nu}\right)$, which arises from a loop and corresponds to an octet-octet-singlet coupling that cannot be described straightforwardly in the framework provided by [18]. Indeed, our generalization of the color-flow representation was prompted by the implementation of the effective Higgs-gluon-gluon vertex in WHIZARD's [22] optimized matrix element generator O'Mega [21]. While it was simple to "fudge" the Feynman rules for a single insertion, it turned out that these prescriptions would give incorrect results for multiple insertions.

This paper is organized as follows: we start with a motivation and description of our implementation of color-flow QCD in section 2 and discuss quantum field-theoretical

\footnotetext{
${ }^{2}$ Independently of Feynman diagrams and quantum field theory, Penrose had earlier introduced a diagrammatical notation for general tensor calculus, including group representations [4]. This approach has evolved into the backbone of an unconventional textbook on the exceptional Lie algebras [5].
} 
aspects in more detail in section 3 . In section 4 , we show that our description is valid to all orders in perturbation theory. In the following two sections we discuss some applications in detail: tree level amplitudes in section 5 and the already mentioned effective interactions from loops in section 6. For reference, we repeat the color-flow Feynman rules for QCD with fermionic matter in the fundamental representation in appendix A. As an example of exotic color representations, we extend those rules to color-sextet particles in appendix B.

\section{The model}

In this section, we construct a variant of QCD which contains two extra gauge degrees of freedom. The model is set up not for its physics content, but for its usefulness in practical perturbative calculations. In fact, as we will show in later sections, in physical quantities the extra degrees of freedom cancel, so all predictions are identical to ordinary QCD.

\subsection{QCD preliminaries}

We consider QCD with a single fermionic matter species in the $N$-dimensional fundamental representation of $\mathrm{SU}(N)$. While we are really interested in the $N=3$ case, it is sometimes useful to explicitly keep the dependence on $N$, as we will do through most of this paper. Furthermore, we will not assume that the matter representation is vector-like as in QCD, although we do follow the QCD notation with Dirac fermions in the Lagrangian.

Let us first recall basic facts of perturbative QCD. The perturbation series is derived from a Lagrangian which splits into two parts,

$$
\mathcal{L}=\mathcal{L}_{\text {inv }}+\mathcal{L}_{\text {gf }}
$$

a gauge-invariant part $\mathcal{L}_{\text {inv }}$ and a gauge-fixing part $\mathcal{L}_{\text {gf }}$. The gauge-invariant part is given by ${ }^{3}$

$$
\mathcal{L}_{\text {inv }}=-\frac{1}{2 g^{2}} \operatorname{Tr} \hat{G}_{\mu \nu} \hat{G}^{\mu \nu}+\bar{\psi}(\mathrm{i} \not \partial+\hat{A}) \psi .
$$

The gauge-fixing part depends on the chosen gauge-fixing procedure. In a manifestly covariant formulation with linear gauge-fixing, it takes the form

$$
\mathcal{L}_{\text {gf }}=\frac{2}{g^{2}} \operatorname{Tr} \hat{B}(\partial \cdot \hat{A})+\frac{\xi}{g^{2}} \operatorname{Tr} \hat{B}^{2}-\frac{2}{g^{2}} \operatorname{Tr} \hat{\bar{c}} \partial^{\mu}\left(\partial_{\mu} \hat{c}-\mathrm{i}\left[\hat{A}_{\mu}, \hat{c}\right]\right) .
$$

Since the matter fields are in the fundamental (defining) $N$-dimensional representation of $\mathrm{SU}(N)$, we choose to represent the Lie-algebra valued fields $G, A, B, c, \bar{c}$ by traceless $N \times N$ matrices. ${ }^{4}$ The field-strength tensor $\hat{G}$ can be expressed in terms of the gauge potential $\hat{A}$ as

$$
\hat{G}_{\mu \nu}=\partial_{\mu} \hat{A}_{\nu}-\partial_{\nu} \hat{A}_{\mu}-\mathrm{i}\left[\hat{A}_{\mu}, \hat{A}_{\nu}\right]
$$

Furthermore, the gauge-fixing term involves a Fadeev-Popov [25] ghost field $\hat{c}$, an antighost field $\hat{\bar{c}}$ and a Nakanishi-Lautrup (NL) [26, 27] auxiliary field $\hat{B}$. The latter can be integrated

\footnotetext{
${ }^{3}$ We set the masses of matter fields to zero, for brevity.

${ }^{4}$ For later convenience, we mark traceless matrix fields by a hat, $\hat{A}_{\nu}$.
} 
out in order to obtain the more familiar form of the gauge fixing Lagrangian

$$
\mathcal{L}_{\text {gf }}=-\frac{1}{g^{2} \xi} \operatorname{Tr}(\partial \cdot \hat{A})^{2}-\frac{2}{g^{2}} \operatorname{Tr} \hat{\bar{c}} \partial^{\mu}\left(\partial_{\mu} \hat{c}-\mathrm{i}\left[\hat{A}_{\mu}, \hat{c}\right]\right) .
$$

We may assume a manifestly gauge-invariant renormalization procedure such as $\overline{\mathrm{MS}}$, so that the Lagrangian retains the form $((2.2),(2.3))$ in each order of the perturbative expansion. ${ }^{5}$ The fields in the Lagrangian are understood to be renormalized, order by order, such that the model contains only two (renormalized) real parameters, the gaugecoupling $g$ and the gauge-fixing parameter $\xi$. The latter drops out of physical quantities.

The gauge group $\mathrm{SU}(N)$ is a subgroup of the general linear group $\mathrm{GL}(N)$, therefore the fields (e.g., $\hat{A}$ ), in the $N \times N$ matrix representation, obey algebraic constraints:

$$
\hat{A}^{\dagger}=\hat{A} \quad \text { and } \quad \operatorname{Tr} \hat{A}=0 .
$$

The constraints are automatically satisfied if we introduce the usual basis for the Lie algebra representation, $T^{a}\left(a=1, \ldots N^{2}-1\right)$, and write $\hat{A}=\sum_{a} A^{a} T^{a}$. The basis elements obey the hermiticity and trace conditions:

$$
\operatorname{Tr} T^{a}=0, \quad \operatorname{Tr} T^{a} T^{b}=\frac{1}{2} \delta^{a b}, \quad\left[T^{a}, T^{b}\right]=i f^{a b c} T^{c}, \quad\left(T^{a}\right)^{\dagger}=T^{a}
$$

with the real structure constants $f^{a b c}$. Each Feynman graph can be factorized into a kinematical and a color amplitude. The color amplitude consists of a string of $T$ and $f$ tensors, contracted over all internal indices, and representable as a combination of tensors with open external indices $a, b, \ldots, i, j, \ldots$. For squared amplitudes, all indices are summed over, hence the particular representation becomes irrelevant. Various schemes exist to compute color amplitudes exactly and efficiently.

\subsection{The color-flow representation}

In the following, we will eliminate the basis $T^{a}$, and instead work with the individual matrix elements of the gauge fields in the chosen Lie algebra representation [5]. This approach is known as a color-flow representation. For concreteness, we consider the gauge potential $\hat{A}$. Without algebraic constraints, a $N \times N$ matrix field contains $N^{2}$ complex degrees of freedom.

We have to implement the algebraic contraints (2.6). The hermiticity condition is

$$
\hat{A}^{i}{ }_{j}=\left(\hat{A}^{*}\right)^{j}{ }_{i}
$$

In effect, each unordered index combination $(i j)$ carries only one, instead of two, complex degree of freedom. We can understand this as if each ordered index combination $(i, j)$ carried one real degree of freedom. For the propagator in a Feynman graph, a value of an index can be represented by a colored line. There are $N$ different colors which correspond to the $N$ values each index may take. An arrow of the line indicates whether the index is the lower or upper one. Hence, $\hat{A}$ propagators carry two color lines with opposing directions.

\footnotetext{
${ }^{5} \mathrm{~A}$ generic renormalization procedure could make non-invariant terms in the renormalized Lagrangian necessary that ensure gauge invariance of the effective action.
} 
Similarly, matter propagators are represented by a single directed color line. External states are represented by a terminating line (matter) or two opposing terminating lines (gauge). As long as we consider only the interactions following from the Lagrangian as quoted above, the rule of matrix multiplication trivially ensures that color is conserved at each vertex, i.e., lines end only at external states.

While this graphical approach is straightforward, we also have to enforce the trace condition from (2.6), explicitly

$$
\sum \hat{A}_{i}^{i}=0
$$

which reduces the number of degrees of freedom by one. The implementation of this constraint amounts to additional modified color-flow patterns in Feynman graphs [18]. While the construction of these patterns is easy to understand, they are, by definition, a nonlocal modification of the naive color-flow expansion. This is a complication in automatic, in particular non-diagrammatic, algorithms, which we want to avoid.

Instead, we follow a field-theoretical approach and set up a modified QCD theory. This theory has the algebraic constraints inherent in its field content, such that they are automatically satisfied by the generated color-flow amplitudes. In essence, it generates (i) terms that complete the $A$ field to a $\mathrm{U}(N)$ matrix so that it has no trace condition and naive color-flow Feynman rules apply, and separately (ii) terms that subtract the spurious contributions without complicating the color-flow rules. To make the diagrammatic expansion unambiguous, the two fields have to be formally independent of each other.

\subsection{Singlet and phantom gluons}

We denote the field which complements the gluon field matrix as the singlet gluon $A_{0}$. This field is associated with a "zeroth" generator $T^{0}$ which is not traceless but satisfies

$$
\operatorname{Tr} T^{0}=\sqrt{\frac{N}{2}}, \quad \operatorname{Tr} T^{a} T^{0}=\frac{1}{2} \delta^{a 0}, \quad\left[T^{a}, T^{0}\right]=0,
$$

and let $a$ run from 0 to $N^{2}-1$. Explicitly,

$$
\left(T^{0}\right)^{i}{ }_{j}=\frac{1}{\sqrt{2 N}} \delta_{j}^{i}
$$

(We choose upper indices for the fundamental and lower indices for the anti-fundamental color representation.) The matrix field resulting from combining $\hat{A}$ with $A_{0}$

$$
A=\hat{A}+A^{0} T^{0}=\left(\sum_{a=0}^{N^{2}-1} A^{a} T^{a}\right)
$$

has $N^{2}$ independent components, the color-flow gluons $A_{j}^{i}$. Analogously, for the NL and ghost fields, we replace the traceless fields by unconstrained fields $B, c, \bar{c}$ and singlet fields $B_{S}, c_{S}, \bar{c}_{S}$, respectively. The field strength $G$ is modified accordingly.

For the subtraction terms, we introduce a phantom gluon field $\tilde{A}$. The phantom gluon is an independent $\mathrm{U}(1)$ gauge boson with the wrong sign in the propagator. Like the other gluons, it couples to the matter fields via the QCD coupling $g$. It does not couple to gluon 
fields. We also introduce the corresponding NL field $\tilde{B}$. (We may also introduce phantom ghost fields, but these can be omitted with linear gauge fixing, since the U(1) gauge group is abelian.)

\subsection{Color-flow QCD}

We can now set up the Lagrangian for the color-flow version of QCD, that includes the singlet gluon (as a component of the gluon matrix $A^{i}{ }_{j}$ ) and the phantom gluon $\tilde{A}$, together with their associated field strength, NL and ghost fields.

The Lagrangian ${ }^{6}$ is

$$
\begin{aligned}
\mathcal{L}= & -\frac{1}{2 g^{2}} \operatorname{Tr} G_{\mu \nu} G^{\mu \nu}+\frac{N}{2 g^{2}} \tilde{G}_{\mu \nu} \tilde{G}^{\mu \nu}+\bar{\psi}(\mathrm{i} \not \partial+A-\tilde{A}) \psi \\
& +\frac{2}{g^{2}} \operatorname{Tr} B(\partial \cdot A)-\frac{2 N}{g^{2}} \tilde{B}(\partial \cdot \tilde{A})+\frac{\xi}{g^{2}} \operatorname{Tr} B^{2}-\frac{\xi N}{g^{2}} \tilde{B}^{2}+\mathcal{L}_{\text {ghost }}
\end{aligned}
$$

where

$$
\mathcal{L}_{\text {ghost }}=-\frac{2}{g^{2}} \operatorname{Tr} \bar{c} \partial^{\mu}\left(\partial_{\mu} c-\mathrm{i}\left[A_{\mu}, c\right]\right)
$$

and the decoupling ghosts for the phantom gluon have been omitted.

A priori, this theory is different from QCD. We will denote it as "color-flow QCD" and we will show its equivalence to QCD in section 3. It contains $N^{2}$ instead of $N^{2}-1$ independent degrees of freedom in the $A$ field, plus the additional $\mathrm{U}(1)^{\prime}$ gauge field $\tilde{A}$ with wrong-sign propagator. Thus, it can be regarded as a $\mathrm{U}(N) \times \mathrm{U}(1)^{\prime}$ or, equivalently, $\mathrm{SU}(N) \times \mathrm{U}(1) \times \mathrm{U}(1)^{\prime}$ gauge theory. In a path-integral formulation, there are independent integrations over all $N^{2}+1$ gauge components. The singlet field $A^{0}$ is present, but hidden in the gauge-field matrix.

In the Lagrangian (2.13), we have encoded the algebraic properties of the color-flow decomposition as a (perturbative) quantum field theory on its own. Therefore, we can expect that amplitudes calculated from this field theory manifestly exhibit the advantages of this approach.

In calculations, we therefore retain the decomposition of the matrix-valued fields in terms of their matrix elements, $A_{j}^{i}$. As explained above, these non-hermitean vector bosons are represented by double lines, one for the color and one for the anticolor flow, flowing in opposite directions. The vertex color factors are Kronecker deltas, which diagrammatically correspond to color lines continuing through the vertex. The phantom gluon, on the other hand, is analogous to a photon. In particular, it carries no color flow. Color lines always terminate at external states. Hence, for any tree diagram, the color factor is simply $N^{p}$, where $p$ is the number of distinct color lines in the diagram (after squaring). Since this is determined by the colors of the external states, the whole color algebra becomes trivial. The

\footnotetext{
${ }^{6}$ For the purpose of discussing renormalization, we incorporate the gauge coupling in the normalization of the gauge-field and gauge-fixing terms. To derive Feynman rules later, we will apply a trivial field renormalization to transfer $g / \sqrt{2}$ to the vertices and obtain a canonically normalized $\mathrm{U}(N)$ gauge field from (2.13). However, we will retain the factor $N$ in the phantom kinetic term and it will appear in the phantom propagator and external states.
} 
price for this is a proliferation of extra Feynman diagrams (which have identical kinematics, however, and thus do not blow up the calculation).

We list the Feynman rules that follow from (2.13) in appendix A.

\subsection{Example}

Let us look at the exchange of a gluon between fermion lines. In ordinary QCD, this is represented by the diagram

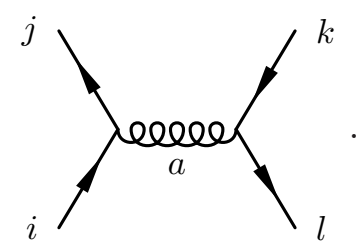

In color-flow QCD, we get two diagrams, one for gluon and one for phantom exchange
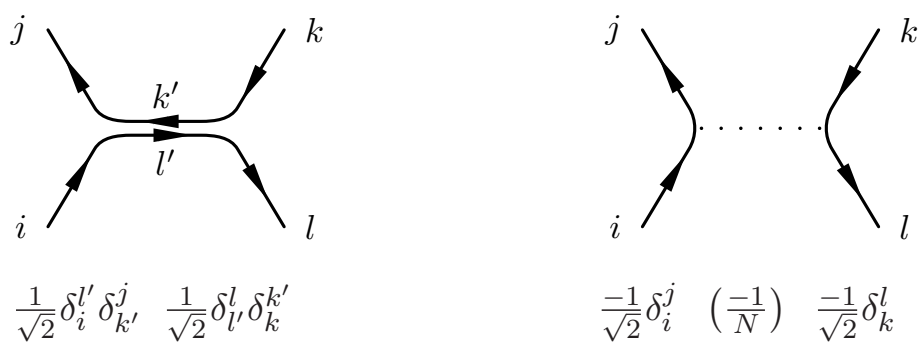

with the correct sum

$$
\frac{1}{2}\left(\delta_{i}^{l} \delta_{k}^{j}-\frac{1}{N} \delta_{i}^{j} \delta_{k}^{l}\right)=\sum_{a}\left(T^{a}\right)_{i}^{j}\left(T^{a}\right)_{k}^{l} .
$$

Usually, this color-flow decomposition is obtained by applying the relation (2.15) backwards. The Feynman rules derived from the color-flow Lagrangian generate it directly.

Also at the squared-amplitude level, the color-flow representation leads to a reorganization of the color-flow calculation, which considerably simplifies the algorithm. There is a further benefit: common parton-shower and hadronization algorithms expect the color connections to be expressed in a color-flow basis. We obtain both the exact amplitude (at the given order) and, by omitting the interference terms and all terms containing external phantoms, the projection on the possible color-flow patterns in a single step.

In summary, we work with a trivial version of color algebra since the complete color flow information is represented on a graphical basis. Subtractions in the color factors are accounted for by extra diagrams which involve the phantom gluon. No approximation is involved.

\section{Field-theoretical considerations}

In the following, we demonstrate that color-flow QCD as described above is equivalent to the original $\mathrm{SU}(N)$ theory, i.e., $A^{0}$ and $\tilde{A}$ cancel each other in physical quantities. This equivalence may appear trivial, at least in tree-level amplitudes. However, it bears some 
subtleties, in particular if it is to be extended to all orders. To promote the approach from a technical device to a consistent field theory, we have to prove that the equivalence can be maintained, by suitable renormalization conditions, to all orders in perturbation theory.

This situation is similar to the covariant quantization of QED, where the scalar and longitudinal photon components, which are both unphysical, cancel in physical quantities. In a nonabelian theory, this requires the additional introduction of ghost fields [25, 28-36]. In the Fock space, the unphysical fields lead to indefinite-norm states, but it is possible to build a Hilbert space out of equivalence classes of physical states in which the $S$ matrix is unitary. As we will show below, the same argument applies in the present case: the phantom gluon generates negative-norm states, but it is always produced coherently with the singlet gluon (which is now hidden in the $N \times N$ gluon matrix), and therefore a unitary $S$ matrix is well defined.

\subsection{Algebraic identities at lowest order}

A consistent formulation of a quantum field theory is based on the symmetries that the effective action, including the integrated Lagrangian as its tree approximation, has to satisfy order by order in a perturbative expansion. These are representable as algebraic identities [35, 36].

In the present subsection, we look only at the lowest order, so we temporarily ignore the complications that necessitate the replacement of gauge symmetry by BRST symmetry, ghost fields, etc. Given the fields $A^{i}{ }_{j}, \tilde{A}, B^{i}{ }_{j}, \tilde{B}, \bar{\psi}, \psi$, we observe that the lowest-order action $\Gamma^{0}=\int \mathcal{L}$ from $(2.13)$, with the ghost term omitted, satisfies the Ward identity ${ }^{7}$ of $\mathrm{U}(N)$,

$$
\operatorname{Tr}\left[(\delta A) \frac{\delta \Gamma}{\delta A}\right]+(\delta \bar{\psi}) \frac{\delta \Gamma}{\delta \bar{\psi}}-\frac{\delta \Gamma}{\delta \psi}(\delta \psi)=\frac{2}{g^{2}} \operatorname{Tr}[B \partial \cdot \delta A]
$$

where the gauge transformations of the fields are

$$
\delta A=\partial \omega-\mathrm{i}[A, \omega], \quad \delta \tilde{A}=0, \quad \delta \psi=\mathrm{i} \omega \psi, \quad \delta \bar{\psi}=-\mathrm{i} \bar{\psi} \omega, \quad \delta B=0 .
$$

This Ward identity decomposes into separate identities for the $\mathrm{SU}(N)$ and $\mathrm{U}(1)$ factors. Even though we are interested only in the U(1) part, we want to keep our notation free of the clutter of indices. Therefore, we retain the matrix notation and use traces to project on the $\mathrm{U}(1)$. Indeed, we can evaluate the Ward identity (3.1) for $\omega=\omega_{0} \mathbf{1}$ to find

$$
\partial_{\mu} \operatorname{Tr} \frac{\delta \Gamma}{\delta A_{\mu}}+\mathrm{i}\left(\bar{\psi} \frac{\delta \Gamma}{\delta \bar{\psi}}+\frac{\delta \Gamma}{\delta \psi} \psi\right)=-\frac{2}{g^{2}} \partial^{2} \operatorname{Tr} B
$$

This is accompanied by the gauge-fixing condition for the singlet

$$
\operatorname{Tr} \frac{\delta \Gamma}{\delta B}=\frac{2}{g^{2}}(\operatorname{Tr} \partial \cdot A+\xi \operatorname{Tr} B) .
$$

\footnotetext{
${ }^{7}$ All functional derivatives are defined to act from the left which introduces a sign for the variation of fermions

$$
\delta \Gamma=\delta \bar{\psi}_{\alpha} \frac{\delta \Gamma}{\delta \bar{\psi}_{\alpha}}+\delta \psi_{\alpha} \frac{\delta \Gamma}{\delta \psi_{\alpha}}=\delta \bar{\psi} \frac{\delta \Gamma}{\delta \bar{\psi}}-\frac{\delta \Gamma}{\delta \psi} \delta \psi
$$
}


Furthermore we define, for any matrix-valued field $M \in\{A, G, \ldots\}$, the linear combinations

$$
M_{ \pm}=\frac{1}{\sqrt{2}}\left(\frac{1}{N} \operatorname{Tr} M \pm \tilde{M}\right)
$$

and the dual functional operators

$$
\frac{\delta \Gamma}{\delta M_{ \pm}}=\frac{1}{\sqrt{2}}\left(\operatorname{Tr} \frac{\delta \Gamma}{\delta M} \pm \frac{\delta \Gamma}{\delta \tilde{M}}\right)
$$

with

$$
\frac{\delta M_{ \pm}(x)}{\delta M_{ \pm}(y)}=\delta^{4}(x-y), \quad \frac{\delta M_{ \pm}(x)}{\delta M_{\mp}(y)}=0 .
$$

Using (3.5), we can rewrite terms appearing in the Lagrangian as

$$
\begin{aligned}
\frac{1}{2} \operatorname{Tr} M^{2}-\frac{N}{2} \tilde{M}^{2} & =\frac{1}{2} \operatorname{Tr} \hat{M}^{2}+N M_{+} M_{-} \\
T^{0} M^{0}-\tilde{M} & =\sqrt{2} M_{-} .
\end{aligned}
$$

Computing the derivative of the lowest-order action with respect to $A$ and $\tilde{A}$ and combining the results, we obtain the phantom equation

$$
\frac{\delta \Gamma}{\delta A_{+\nu}}=\frac{2 N}{g^{2}}\left(\partial_{\mu} G_{-}^{\mu \nu}-N \partial^{\nu} B_{-}\right) .
$$

It expresses the cancellation between singlet and phantom terms. Since $A_{+}$is an abelian gauge field, applying $\delta / \delta A_{+}^{\nu}$ to the ghost term (2.14) yields zero, so the phantom equation holds for the complete lowest-order Lagrangian in the BRST formalism [28-31].

\section{$3.2 \quad$ Fock-space cancellation}

To clarify the physical implications of the phantom equation (3.8), let us use (3.7) to express the singlet and phantom fields $\left(\frac{1}{N} \operatorname{Tr} A, \tilde{A}\right)$ by $\left(A_{+}, A_{-}\right)$. The remainder of the matrix $A$ is traceless, this is the original $\mathrm{SU}(N)$ gluon with all of its interactions. We must show that the extra fields $A_{+}$and $A_{-}$do not contribute to observable quantities.

We eliminate the $B$ fields via their equations of motion. The terms in the resulting Lagrangian that depend on $A_{+}$or $A_{-}$take the form

$$
\mathcal{L}=-\frac{N}{g^{2}} G_{+\mu \nu} G_{-}{ }^{\mu \nu}-\frac{2 N}{\xi g^{2}}\left(\partial \cdot A_{+}\right)\left(\partial \cdot A_{-}\right)+\sqrt{2} \bar{\psi}\left(A_{-}\right) \psi .
$$

The propagator interchanges $A_{+}$and $A_{-}$, but only $A_{-}$couples to matter. Couplings to ordinary gluons are absent in (3.9), because $G_{ \pm}^{\mu \nu}=\partial^{\mu} A_{ \pm}^{\nu}-\partial^{\nu} A_{ \pm}^{\mu}$. As a result, whenever a singlet/phantom gluon is created by a matter current, it cannot be annihilated, and vice versa. In short, $A_{+}$and $A_{-}$do not introduce any observable interactions.

In fact, we may freely add any term to the effective action that depends on $A_{-}$at least linearly, but has no dependence on $A_{+}$. Any such interaction is unobservable.

This pattern will continue to hold to all orders, unless a loop diagram induces an interaction of some current with $A_{+}$. However, the phantom equation (3.8) prohibits 
this: it tells that the interactions of $A_{+}$are completely accounted for by the leading-order Lagrangian, up to a renormalization of the coupling constant. Therefore, if we can maintain the phantom equation on the effective action to all orders, observables computed from the color-flow QCD theory are identical to those from ordinary QCD.

In actual applications, we do not express the Lagrangian in terms of $A_{+}$and $A_{-}$. Instead, we work with the $\mathrm{U}(N)$ field $A$ and the $\mathrm{U}(1)^{\prime}$ phantom $\tilde{A}$, as we $\operatorname{did}$ in the previous sections. Hence, the Fock-space cancellation is not entirely trivial since it involves cancellations among graphs that belong to different gauge groups, at least superficially. We have to enforce the phantom equation explicitly in order to guarantee that no $A_{+}$ interaction arises in the effective action.

\subsection{Symmetries}

If the phantom equation (3.8) is combined with the Ward identity (3.3) and the gaugefixing condition (3.4), we obtain an analogous Ward identity and gauge-fixing condition for the phantom gluon. The complete symmetry, at lowest order, is $\mathrm{U}(N) \times \mathrm{U}(1)^{\prime}$, with the additional condition that all gauge couplings are equal (to the $\mathrm{SU}(N)$ gauge coupling).

In the previous subsection, we introduced linear combinations of the extra gauge fields, $A_{+}$and $A_{-}$. Correspondingly, we may identify gauge groups $\mathrm{U}(1)_{+}$and $\mathrm{U}(1)_{-}$which are orthogonal combinations of the original extra $\mathrm{U}(1)$ and $\mathrm{U}(1)^{\prime}$ gauge groups. These gauge invariances are described by the Ward identities

$$
\begin{aligned}
\partial^{\nu} \frac{\delta \Gamma}{\delta A_{-}^{\nu}}+\mathrm{i} \sqrt{2}\left(\bar{\psi} \frac{\delta \Gamma}{\delta \bar{\psi}}+\frac{\delta \Gamma}{\delta \psi} \psi\right) & =-\frac{2 N}{g^{2}} \partial^{2} B_{+}, \\
\partial^{\nu} \frac{\delta \Gamma}{\delta A_{+}^{\nu}} & =-\frac{2 N}{g^{2}} \partial^{2} B_{-},
\end{aligned}
$$

which follow from the Ward identity (3.3) and the phantom equation (3.8).

The corresponding gauge-fixing conditions are

$$
\frac{\delta \Gamma}{\delta B_{ \pm}}=\frac{2 N}{g^{2}}\left(\partial \cdot A_{\mp}+\xi N B_{\mp}\right) .
$$

Note that the matter fields are neutral under $\mathrm{U}(1)_{+}$. This is in line with the observation that $A_{+}$does not interact at all.

We will demonstrate in section 4 that interactions of $A_{+}$are forbidden in the effective action to all higher orders, by the phantom equation, and there is a $U(1)_{+}$symmetry. On the other hand, regarding $\mathrm{U}(1)_{-}$, there is no guarantee that this symmetry can be enforced to all orders. If the gauge representation of the matter fields is chiral (QCD happens to be

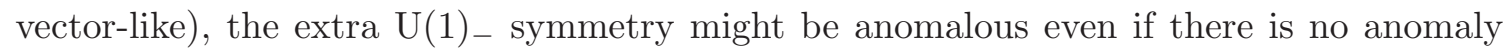
of the original $\mathrm{SU}(N)$. However, by the argument in the previous subsection, we see that a $\mathrm{U}(1)_{-}$anomaly will not contribute to observables, so it does not invalidate unitarity of the $S$ matrix projected onto physical states.

\section{Renormalization}

In this section we outline a proof that the color-flow version of QCD can be extended consistently to all orders in perturbation theory. We follow the algebraic renormalization 
procedure, as explained in detail in ref. [35, 36]. Algebraic renormalization is mainly useful for proving the perturbative renormalizability to all orders of a class of models, in particularly for demonstrating the absence of anomalies, i.e. that symmetries can be maintained in the quantized theory.

It is convenient in practical loop calculations to employ a regularization that maintains the rigid and gauged symmetries of a Lagrangian in order to reduce the number of required counterterms. For recursive proofs to all orders, on the other hand, it is important to prove the absence of divergencies that would require the introduction of counterterms which would break a symmetry of the $n$-loop effective action. For this purpose one can use a non-invariant regulator for which the model-independent quantum action principles [3743] have been established, which limit the possible counterterms to a finite set that can be enumerated explicitly.

The inductive proof of renormalizability proceeds then in three stages: first one derives a set of Slavnov-Taylor (ST) identities for the effective action from the rigid and gauged symmetries of the model under consideration (cf. section 4.1). Subsequently, one proves that the tree-level effective action (i.e., the Lagrangian integrated over space-time) is the unique solution of these functional equations (cf. section 4.2), up to field and coupling constant renormalizations. This establishes an induction hypothesis. For the induction step at $n$-loop order, one shows recursively that these ST identities can be maintained in order $n+1$ of perturbation theory through a suitable choice of local counterterms if they are satisfied by the $n$-loop effective action (cf. section 4.3).

The induction step consists of several parts. One first restricts the terms that can possibly violate the ST identities at order $n+1$, assuming that they hold at order $n$. The quantum action principles guarantee that these terms take the form of local operators with well-defined dimension and quantum numbers, and the ST identities impose further algebraic constraints. The coefficients of the local operators can be computed as the values of certain Feynman graphs. If there are operators with nonvanishing coefficients, one has to check whether they can be cancelled by adding suitable non-invariant counterterms to the Lagrangian. This is a purely algebraic problem. If there is a solution, the ST identities can be restored by the counterterms and renormalizability is proved. Otherwise, the symmetry is manifestly broken by an anomaly.

\subsection{Conditions imposed on the effective action}

First, we need a precise (perturbative) definition of the quantum field theory. We define the effective action of color-flow QCD as a solution of the usual ST identity for $\mathrm{SU}(N)$, here extended to $\mathrm{U}(N)$ :

$$
\int d^{4} x\left(\operatorname{Tr}\left[\frac{\delta \Gamma}{\delta \rho_{A}} \frac{\delta \Gamma}{\delta A}\right]+\frac{\delta \Gamma}{\delta \rho_{\psi}} \frac{\delta \Gamma}{\delta \bar{\psi}}+\frac{\delta \Gamma}{\delta \psi} \frac{\delta \Gamma}{\delta \bar{\rho}_{\psi}}+\operatorname{Tr}\left[\frac{\delta \Gamma}{\delta \rho_{c}} \frac{\delta \Gamma}{\delta c}\right]+\operatorname{Tr}\left[B \frac{\delta \Gamma}{\delta \bar{c}}\right]\right)=0
$$

with the linear gauge-fixing equation

$$
\frac{\delta \Gamma}{\delta B}=\frac{2}{g^{2}}(\partial \cdot A+\xi B) .
$$


These identities involve the gauge, gauge-fixing, and ghost fields $A_{j}^{i}, B_{j}^{i}, c_{j}^{i}, \bar{c}_{j}^{i}$, expressed in the color-flow basis, the matter fields $\psi, \bar{\psi}$, and the sources for the BRST variations, $\rho_{A}, \rho_{\psi}, \bar{\rho}_{\psi}, \rho_{c}$.

Furthermore, the effective action depends on the phantom field $\tilde{A}$ and its associated NL field $\tilde{B}$. The dependence on those fields is accounted for by the phantom equation (3.8), which we state here in the form

$$
\operatorname{Tr} \frac{\delta \Gamma}{\delta A^{\nu}}+\frac{\delta \Gamma}{\delta \tilde{A}^{\nu}}-\frac{2}{g^{2}}\left(\partial^{\mu} \operatorname{Tr} G_{\mu \nu}-N \partial^{\mu} \tilde{G}_{\mu \nu}\right)+\frac{2}{g^{2}}\left(\partial_{\nu} \operatorname{Tr} B-N \partial_{\nu} \tilde{B}\right)=0,
$$

and by the gauge-fixing condition for $\tilde{A}$,

$$
\frac{\delta \Gamma}{\delta \tilde{B}}=-\frac{2 N}{g^{2}}(\partial \cdot \tilde{A}+\xi \tilde{B}) .
$$

The algebraic conditions are supplemented by renormalization conditions which fix the field normalizations and a minimal set of interaction parameters. In the present model, these are just the gauge couplings.

Alternatively, in a minimal-subtraction scheme such as $\overline{\mathrm{MS}}$, we read off renormalization conditions after regularizing the loop integrals and subtracting the divergencies. This can also be done here, but with a caveat: we may use any renormalization scheme for the $\mathrm{SU}(N)$ coupling and for the ordinary $\mathrm{SU}(N)$ fields, but in order to keep the simplicity of the approach, we impose the condition that the singlet $\mathrm{U}(1)$ coupling is equal to the $\mathrm{SU}(N)$ coupling and that the field normalizations are identical. (The ST identity does not enforce this condition since $\mathrm{U}(N)$ is not simple.) For the $\mathrm{U}(1)^{\prime}$ coupling and the phantom field, everything is then fixed by the phantom equation and the phantom gauge-fixing condition.

\subsection{Lowest-order solution}

The next step in the renormalization procedure is to identify the lowest-order effective action. We can verify that the Lagrangian (2.13) is the unique solution of the constraints of the preceding section (with correct gauge group and representation) which is an integrated local polynomial in the fields of dimension equal to four.

The BRST invariance of the theory, and of the lowest-order approximation in particular, is encoded in the ST identity (4.1) and leads to lowest-order BRST transformations of the form

$$
\begin{aligned}
& s A=\partial c-\mathrm{i}[A, c] \quad s c=\mathrm{i} c c \quad s \bar{c}=B \quad s B=0 \\
& s \psi=i c \psi \quad s \bar{\psi}=\mathrm{i} \bar{\psi} c .
\end{aligned}
$$

The U(1) part decouples. The corresponding ghost fields $\operatorname{Tr} c$ and $\operatorname{Tr} \bar{c}$ are free and can be dropped, such that this part of the ST identity can be replaced by the U(1) Ward identity (3.3).

\subsection{Inductive renormalization}

For an inductive proof of renormalizability, we can now assume that we have found a Lagrangian which generates a renormalized effective action at order $n$ that satisfies all 
conditions. The renormalized order- $n+1$ effective action does not necessarily have this property, yet.

We do not repeat the standard derivation and just state that the $\mathrm{SU}(N)$ ST identity (4.1) and gauge-fixing condition (4.2) can be extended to order $n+1$, possibly by adding non-invariant local counterterms to the Lagrangian. Clearly, we can handle the gauge-fixing condition (4.4) in analogy to the $\mathrm{SU}(N)$ gauge-fixing condition, so this equation can also be established at order $n+1$.

The only non-standard part is the phantom equation (4.3). We do not know whether the right-hand side is zero at order $n+1$. The quantum action principles [37-41] tell us, however, that the equation must take the form

$$
\operatorname{Tr} \frac{\delta \Gamma^{(n)}}{\delta A^{\nu}}+\frac{\delta \Gamma^{(n)}}{\delta \tilde{A}^{\nu}}-\frac{2}{g^{2}}\left(\partial^{\mu} \operatorname{Tr} G_{\mu \nu}-N \partial^{\mu} \tilde{G}_{\mu \nu}\right)+\frac{2}{g^{2}}\left(\partial_{\nu} \operatorname{Tr} B-N \partial_{\nu} \tilde{B}\right)=X_{\nu}^{(n+1)},
$$

where the effective action $\Gamma^{(n)}$ is evaluated from the order- $n$ Lagrangian, including terms in the result up to order $n+1$, i.e., diagrams with $n+1$ loops. The operator $X_{\nu}^{(n+1)}$ on the right-hand side is a local polynomial in fields and derivatives of dimension three with the same quantum numbers as the left-hand side.

Taking the divergence of (4.6), we obtain

$$
\partial^{\nu} \operatorname{Tr} \frac{\delta \Gamma^{(n)}}{\delta A^{\nu}}+\partial^{\nu} \frac{\delta \Gamma^{(n)}}{\delta \tilde{A}^{\nu}}+\frac{2}{g^{2}}\left(\partial^{2} \operatorname{Tr} B-N \partial^{2} \tilde{B}\right)=\partial^{\nu} X_{\nu}^{(n+1)} .
$$

This is precisely the Ward identity for the $\mathrm{U}(1)_{+}$symmetry (3.11) with an anomaly on the right-hand side. We have to verify that the right-hand side is in fact zero.

It is well known that the only possible obstruction to such a U(1) Ward identity is the Adler-Bell-Jackiw (ABJ) anomaly [14, 15], i.e., an operator corresponding to a triangle diagram with three external gauge bosons. In a vector-like theory such as QCD, this vanishes. Nevertheless, we may consider a chiral $\mathrm{SU}(N)$ theory where this is not necessarily the case.

With the hypothesis that the phantom equation (4.3) is valid for the renormalized fields and interactions at order $n$, we can apply the change of basis (3.5) to the order- $n$ renormalized fields and rewrite this equation as

$$
\frac{\delta \Gamma^{(n)}}{\delta A_{+\nu}^{(n)}}-\frac{2 N}{g^{2}}\left(\partial_{\mu} G_{-}^{(n) \mu \nu}-\partial^{\nu} \operatorname{Tr} B_{-}^{(n)}\right)=0,
$$

in complete analogy with the tree-level equation (3.8). Taking the second derivative with respect to matter fields of this relation, we immediately see that $A_{+}^{(n)}$ does not interact at all. In particular, the interaction $\left(\bar{\psi} A_{+} \psi\right)^{(n)}$ vanishes. Hence, there is no triangle diagram involving $A_{+}$that can contribute to the ABJ anomaly. Integrating this zero, we conclude that the right-hand side of (4.7) vanishes at order $n+1$. The $\mathrm{U}(1)_{+}$symmetry is preserved. $^{8}$

\footnotetext{
${ }^{8}$ Actual calculations are carried out in the original basis $(A, \tilde{A})$. In this basis, the argument implies exact cancellation between the integrands of loop graphs involving $A$ and $\tilde{A}$. This is analogous to the Standard Model, where the absence of anomalies is evident in the gauge basis $\left(W^{ \pm 0}, B\right)$, but involves a similar relation between $\gamma$ and $Z$ interactions in the physical basis $W^{ \pm}, Z, \gamma$.
} 
Since the right-hand side of (4.7) vanishes, we have $\partial^{\nu} X_{\nu}^{(n+1)}=0$. A divergenceless vector must be the derivative of an antisymmetric tensor, thus

$$
X_{\nu}^{(n+1)}=\partial^{\mu} Y_{\mu \nu}^{(n+1)}
$$

$Y^{(n+1)}$ is a $\mathrm{SU}(N)$-invariant tensor of dimension two, a local polynomial of the fields. The only possibility is

$$
Y_{\mu \nu}^{(n+1)}=a^{(n+1)} \operatorname{Tr} G_{\mu \nu}+b^{(n+1)} \tilde{G}_{\mu \nu}
$$

with constants $a^{(n+1)}$ and $b^{(n+1)}$. If we now modify the Lagrangian by the finite counterterms

$$
\Delta \mathcal{L}=\frac{1}{2} a^{(n+1)} \operatorname{Tr} G_{\mu \nu} \operatorname{Tr} G^{\mu \nu}+\frac{1}{2} b^{(n+1)} \tilde{G}_{\mu \nu} \tilde{G}^{\mu \nu},
$$

and re-evaluate eq. (4.7), this term is cancelled.

In summary, by adding local counterterms to the Lagrangian, we are able to satisfy all required conditions to order $n+1$. In particular, we establish the vanishing of all nontrivial interactions of $A_{+}$, i.e., the exact cancellation of the singlet and phantom interactions.

We note that the cancellation does not apply to the orthogonal combination $A_{-}$, so in the presence of chiral matter, an anomaly operator involving $A_{-}$is allowed. However, as argued in section 3.2 , as long as $A_{+}$does not interact, an operator which involves $A_{-}$,

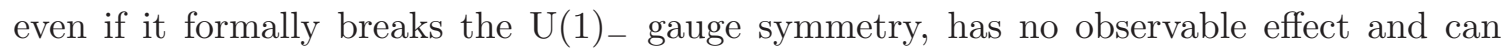
be ignored.

This completes the inductive proof of renormalizability. Unitarity of the $S$-matrix is then established to all orders by the ST identity and by the phantom equation, as argued in section 3.2.

In practice, adding those counterterms is necessary and natural. In the usual $\overline{\mathrm{MS}}$ renormalization scheme, the singlet gluon propagator will receive a renormalization different from the octet gluon propagator, because only the octet has self-couplings. In order to keep the color-flow scheme simple, we require finite counterterms that restore the equality of the propagator residues, and thus of the $\mathrm{SU}(N)$ and $\mathrm{U}(1)$ couplings, at the next order. Then, we also have to renormalize the phantom propagator, i.e., the $\mathrm{U}(1)^{\prime}$ coupling, to the very same value. This renormalization coincides with (4.11). We can do this freely since the values of the $\mathrm{U}(1)$ and $\mathrm{U}(1)^{\prime}$ couplings do not enter any observable quantity.

\section{Applications: tree-level amplitudes}

The color-flow approach is particularly useful for the automatic calculation of tree-level (squared) amplitudes. In this section, we choose a few simple examples that show how this works in practice.

\subsection{Algorithm}

For any amplitude, we replace the QCD diagrams with quarks and gluons by corresponding color-flow diagrams. These contain in place of each octet gluon, either a $\mathrm{U}(N)$ gluon (double color line) or a phantom (no color). The latter appears only in places where the gluon 
directly connects two fermion (i.e., single-color) lines. Each vertex gets a factor $1 / \sqrt{2}$ due to the different normalization of the QCD coupling. After squaring the amplitude, the color lines of each contributing color-flow diagram are connected to the lines of the interfering complex-conjugated diagram, both for the incoming and the outgoing state.

Instead of starting with ordinary QCD diagrams, an implementation may construct the amplitude diagrammatically from scratch, treating $\mathrm{U}(N)$ gluons and quarks of definite color and phantoms as distinct, ordinary particle species. The cross section is computed by squaring diagrams, including all interference terms. We recall that $\mathrm{U}(N)$ gluons do not interfere with phantom gluons.

Each squared or interference diagram has a color weight $W$ which is simply a signed integer power of $N$, given by

$$
W=N^{L}\left(\frac{-1}{N}\right)^{I+E}
$$

where

$$
\begin{aligned}
L & =\text { Number of distinct closed color lines } \\
I & =\text { Number of internal phantom propagators } \\
E & =\text { Number of external phantom particles }
\end{aligned}
$$

The $N^{L}$ factor originates from contracting each string of Kronecker deltas, which represent color conservation at vertices, to a single $\delta_{i}^{i}=N$. The $-1 / N$ factors in the phantom propagators, including those across the cuts, result directly from the Lagrangian.

It is possible to absorb the $(-1 / N)^{I}$ factor for internal lines by including $-1 / N$ in the phantom propagator, as done in the Feynman rules in appendix A. The other factors are applied after squaring the amplitude. Alternatively, we could absorb all $1 / N$ factors, but not their signs, in the phantom-fermion coupling.

In automatic calculations, the color-flow approach has the advantage that the combinatorics of constructing Feynman graphs is already implemented, so the proliferation of diagrams does not raise a bookkeeping problem. (The algorithm should avoid to compute identical kinematics twice, however.) Counting distinct colors is rather simple and can be done, for tree graphs, by looking at the external state. In fact, common conventions $[16,17]$ require an event-generating program to classify the external state in terms of color connections. On the other hand, computing color factors algebraically in a $T^{a}-f^{a b c}$ basis requires some additional infrastructure, and the transformation to the color-connection basis has to be done explicitly.

Algorithms that do not expand an amplitude in diagrams but compute off-shell wave functions recursively [19-21] benefit even more from the color-flow approach. Assigning color factors to diagrams is not applicable there, so a straightforward implementation of QCD color algebra would require keeping a color degree of freedom in each off-shell wave function while constructing the amplitude, and applying a color-matrix multiplication at each vertex. By contrast, in the color-flow approach each color line can be understood as labeling an independent particle species, hence the algorithm need not know about color at all. It just has to distinguish particle species and their respective Feynman rules. 
In the following examples, we verify the color-flow result against the equivalent coloralgebra result, where we evaluate the appropriate trace of $T^{a}$ and $f^{a b c}$ matrices directly. We are not interested in the kinematical part of the calculation that involves propagators, Lorentz factors, and integrations over momenta, so we omit them. We also leave out the QCD coupling which is attached to each vertex, and the color-averaging factor for the initial state. We just quote color weights.

\subsection{Quark-antiquark scattering}

We are looking at the process $\left(q \neq q^{\prime}\right)$

$$
q \bar{q}^{\prime} \rightarrow q \bar{q}^{\prime}
$$

in pure QCD, so at tree level there is a single diagram, gluon exchange.

To eliminate all open color indices, we square the amplitude and sum over colors. In the diagrams, squaring connects the final state of the amplitude and its complex conjugate; this is indicated by a vertical dashed line. Similarly, the initial states of the amplitude and its complex conjugate, the open lines at the left and right margins are also understood to be pairwise connected.
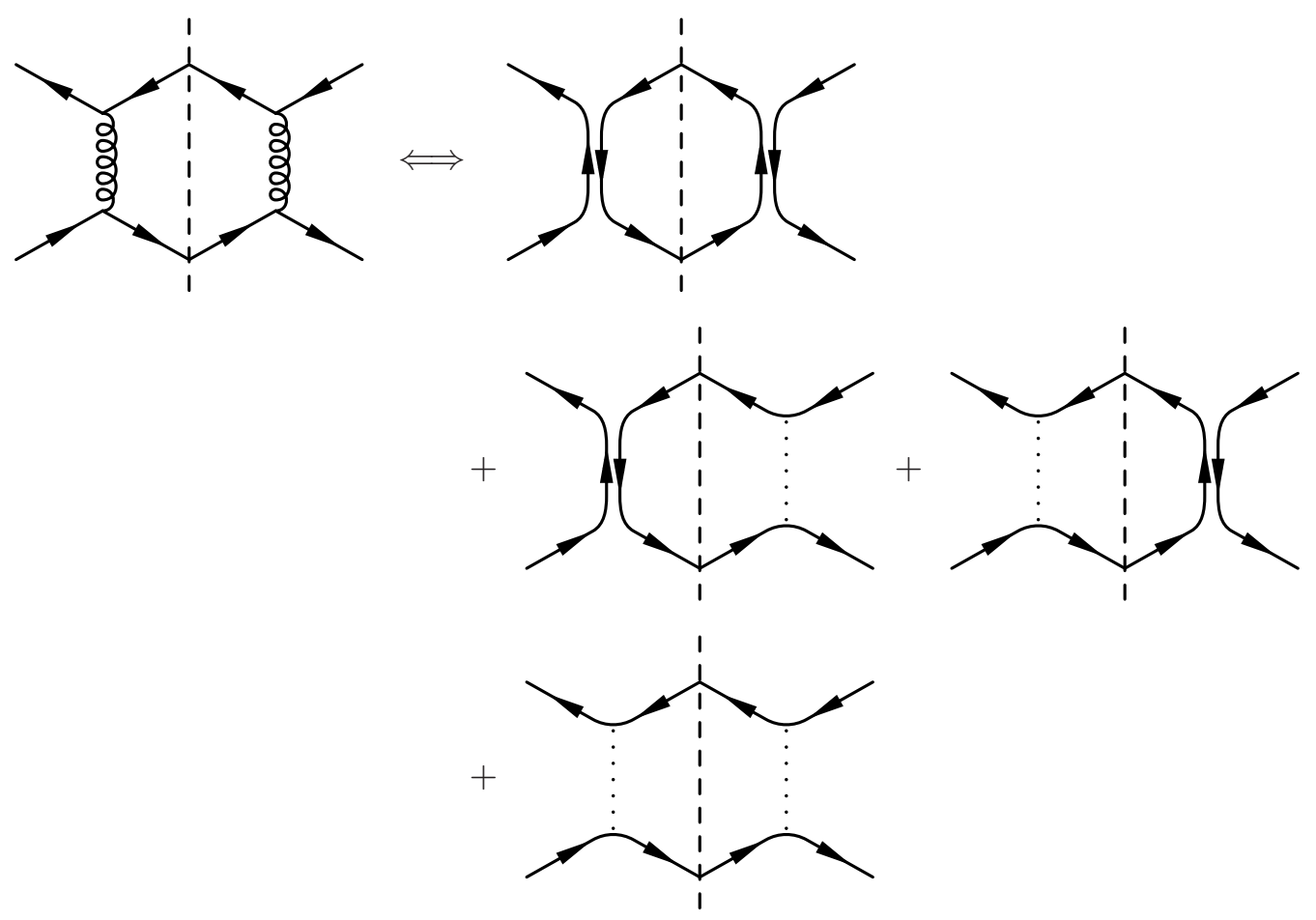

For this squared amplitude, standard color algebra yields

$$
W=\operatorname{Tr}\left[T^{a} T^{b}\right] \operatorname{Tr}\left[T^{a} T^{b}\right]=\frac{1}{4} \delta^{a b} \delta^{a b}=\frac{1}{4}\left(N^{2}-1\right)
$$

as its color weight. 
Color-flow QCD gives four diagrams for the squared amplitude, which differ only in the color factor. We recall that there is a factor $N$ for each closed color line, and a factor $-1 / N$ for each phantom propagator. Including $1 / \sqrt{2}$ for each vertex, we obtain

$$
W=\frac{1}{4}\left(N^{2}+N\left(\frac{-1}{N}\right)+N\left(\frac{-1}{N}\right)+N^{2}\left(\frac{-1}{N}\right)^{2}\right)=\frac{1}{4}\left(N^{2}-1\right)
$$

We observe that there is some redundancy in this case which could be eliminated before computing the result. In any case, we can read off the color factor directly without using color algebra.

\section{3 $\quad$ Four-jet production in $e^{+} e^{-}$}

Let us now consider

$$
e^{+} e^{-} \rightarrow q \bar{q} g g
$$

where we can ignore the colorless initial state when drawing color-flow diagrams. For the amplitude, we have three QCD diagram structures which decompose into ten color-flow diagrams:

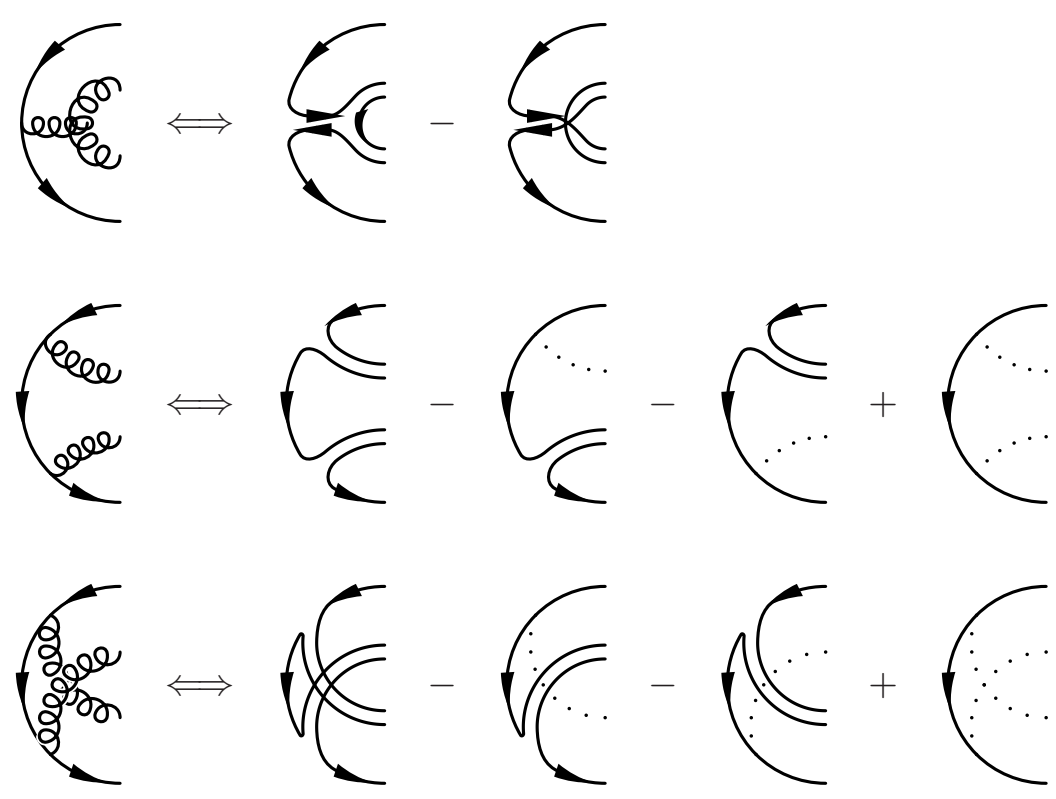

We now look at the squared color-flow diagrams. Symmetries between diagrams simplify the calculation. Summing over all diagrams with common kinematics, we recover the QCD color weights as linear combinations of powers of $N$. We show three terms, the remaining 
ones are equivalent regarding their color flow:
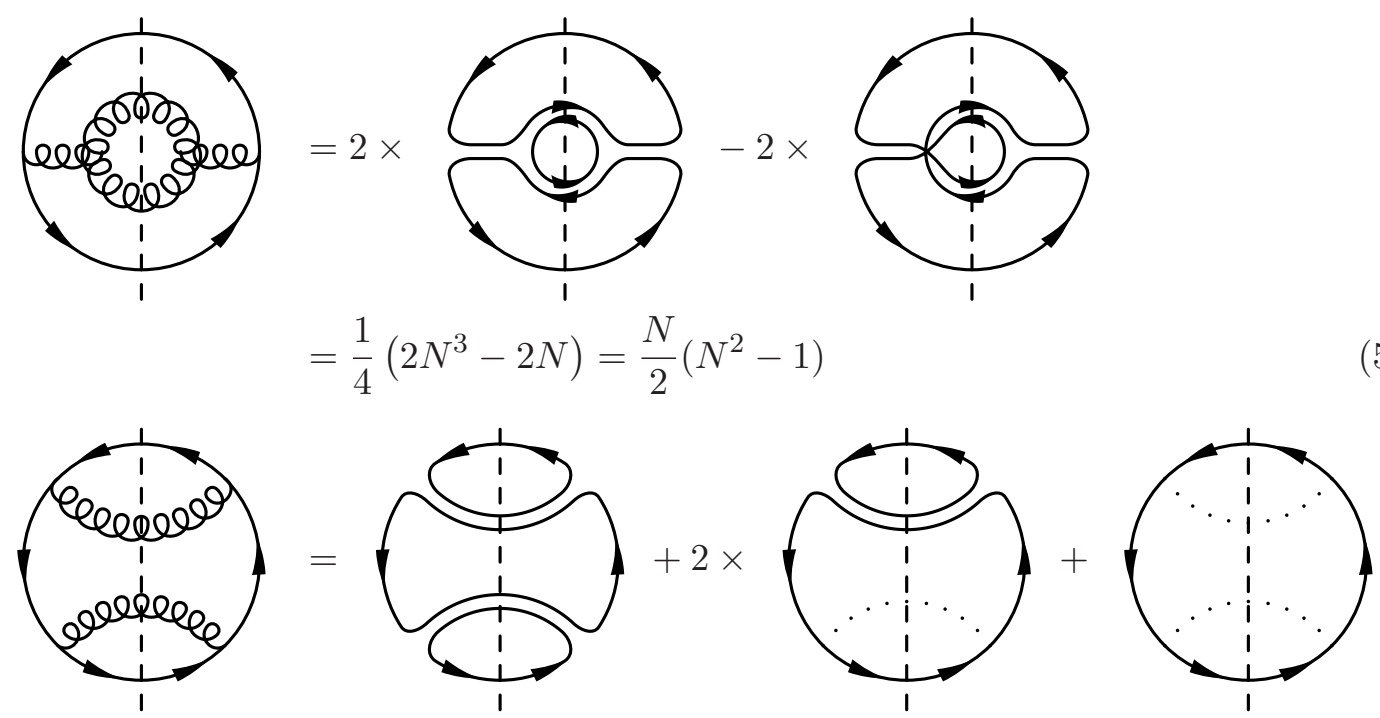

$=\frac{1}{4}\left(N^{3}+2 N^{2}\left(\frac{-1}{N}\right)+N\left(\frac{-1}{N}\right)^{2}\right)=\frac{N}{4}\left(N-\frac{1}{N}\right)^{2}$
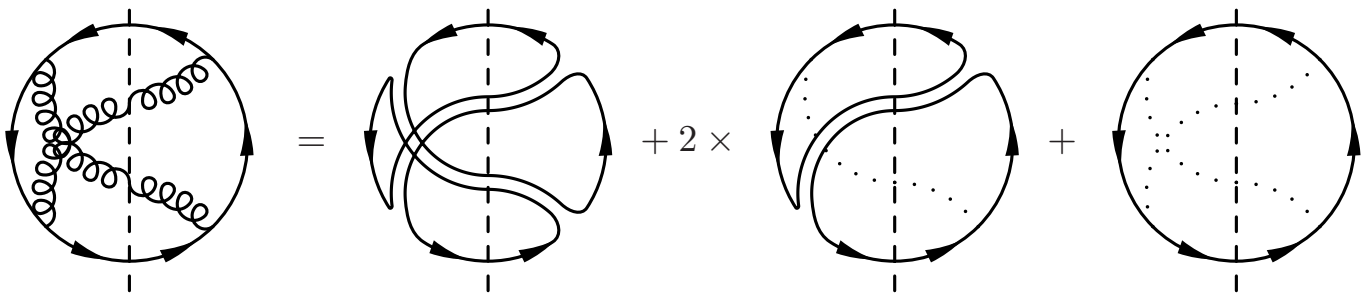

$$
=\frac{1}{4}\left(N+2 N^{2}\left(\frac{-1}{N}\right)+N\left(\frac{-1}{N}\right)^{2}\right)=-\frac{1}{4}\left(N-\frac{1}{N}\right)
$$

In the color-algebra formalism, we compute the color weights

$$
\begin{aligned}
-\operatorname{Tr}\left[T^{a} T^{b}\right] f^{a d c} f^{b c d} & =\frac{N}{2}\left(N^{2}-1\right), \\
\operatorname{Tr}\left[T^{a} T^{a} T^{b} T^{b}\right] & =\frac{N}{4}\left(N-\frac{1}{N}\right)^{2}, \\
\operatorname{Tr}\left[T^{a} T^{b} T^{a} T^{b}\right] & =-\frac{1}{4}\left(N-\frac{1}{N}\right),
\end{aligned}
$$

which agree with the color-flow results, as required.

\section{Applications: effective interactions from loops}

At tree level, the color-flow method expresses all amplitudes in terms of Feynman rules that exclusively contain $\mathrm{U}(N)$ gluons and phantom gluons. This allows us to compute the color factor of any color-flow diagram in the expansion of a squared tree-level amplitude by merely counting the number of distinct external color-flow lines, as detailed in the 
previous section. The factors for the internal phantom propagators are accounted for in the normalization of the propagators.

Once loop diagrams are involved, this is no longer true. Obviously, there may be closed color loops not attached to external lines. As a further complication, there is a particular class of loop (sub-)diagrams where singlet gluons make their appearance, distinguished from the two classes of $\mathrm{U}(N)$ and phantom gluons that we encounter at tree level. Fortunately, this only slightly complicates the algorithm, and computing color factors remains straightforward.

We are particularly interested in loop amplitudes that can be inserted as effective vertices in tree-level diagrams. We absorb the color sum over internal closed color lines in the corresponding vertex factor. As a result, we again can deduce the remaining overall color factor of any amplitude merely looking at the external lines.

An important example is the coupling of Higgs and electroweak bosons to gluons, which occurs first at one-loop level. As in the previous section, we are interested only in color flow, so we ignore all kinematical and coupling factors.

\section{1 $g g \rightarrow H$}

Let us consider colorless particles (in particular, the Higgs boson) coupled to a gluon pair via a fermion (more generically, matter) loop. From the color flows in the triangle diagram

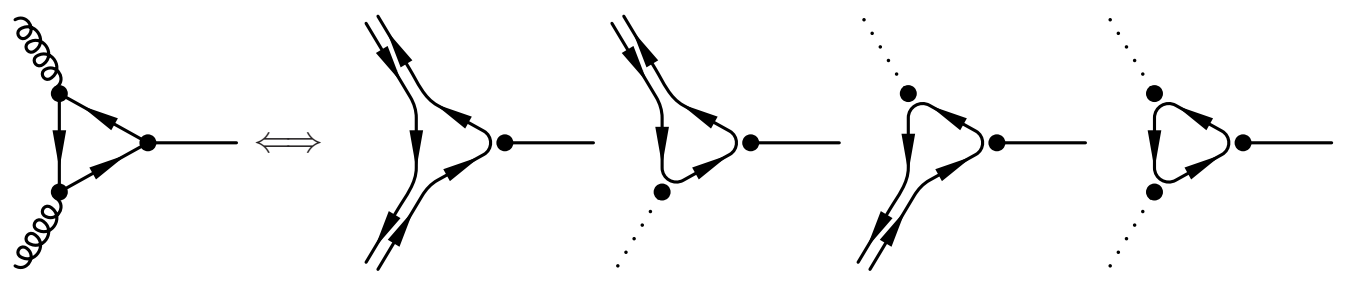

we can derive a set of equivalent "effective" Feynman rules
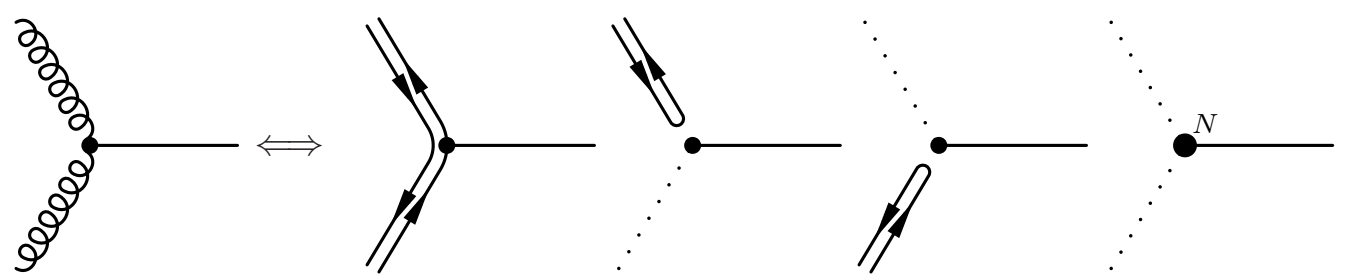

Thus, in the resulting one-loop effective action there appear vertices that explicitly couple phantom gluons to singlet gluons (and to themselves). In the effective action of pure QCD, this is not the case. Graphically, we identify the singlet, the projection of a $\mathrm{U}(N)$ gluon, as a color line being reflected at the vertex. Note that there is no Feynman rule

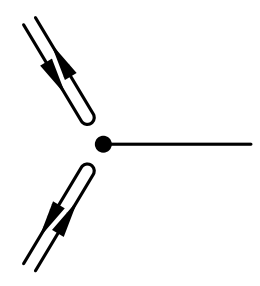


because the singlet-singlet interaction is incorporated in the vertex where the color lines pass through.

These new Feynman rules for the effective vertex yield the correct result for $H \rightarrow g g$ and, equivalently, $g g \rightarrow H$. Squaring the amplitude, we get the set of squared diagrams (external Higgs lines not drawn):

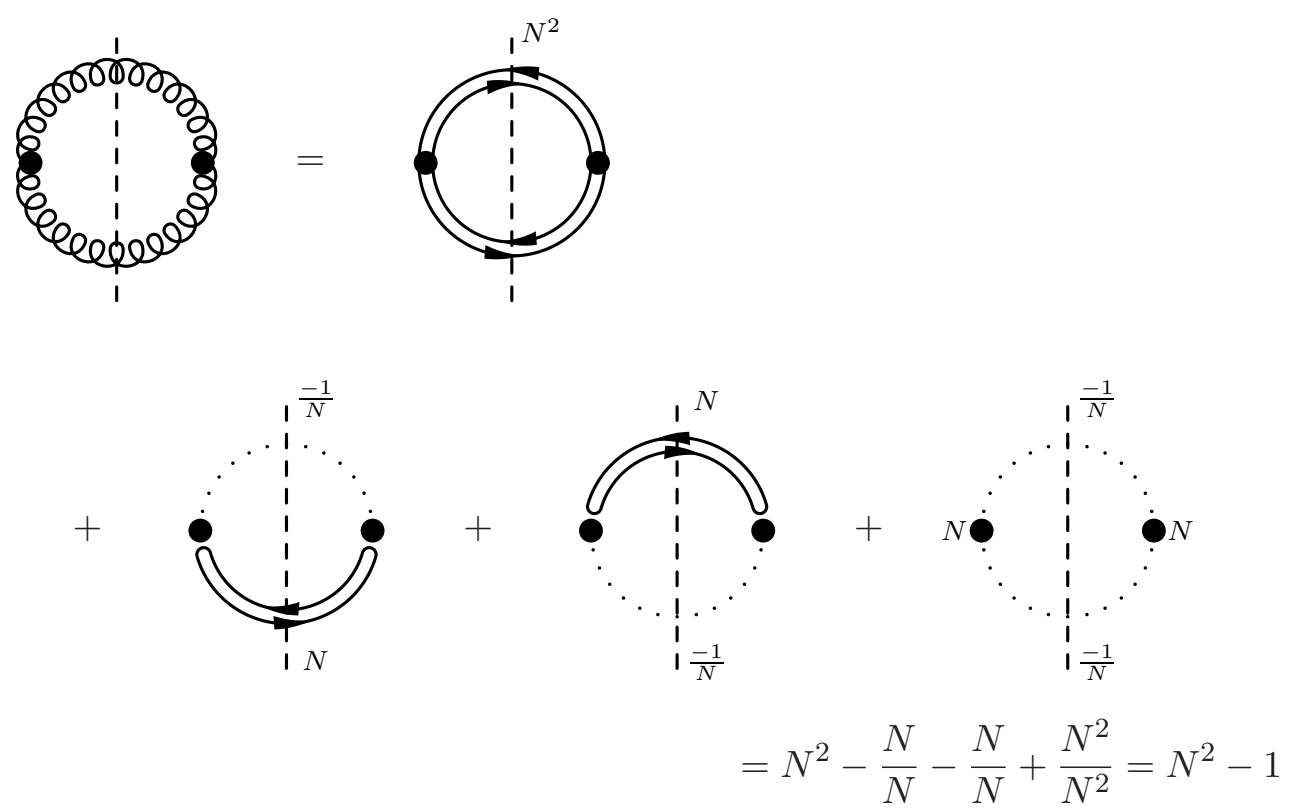

Here, there are no interferences. To get the correct factors, we only have to remember that each phantom gluon comes with a factor $-1 / N$, while the singlet gluon carries color, which yields a factor $N$ when summed over. As in the tree-level case, we just have to count color lines crossing the cut to obtain the color weights of the squared diagrams.

\section{2 $g g \rightarrow H H$}

Things become interesting when there can be two loop-induced effective vertex insertions, as in the process $g g \rightarrow H H$. (The irreducible effective $g g H H$ vertex has the same color structure as $g g H$, so we do not consider it here.) In this case, singlet gluons interfere with $\mathrm{U}(N)$ gluons, projecting out the singlet part on the other side of the cut:
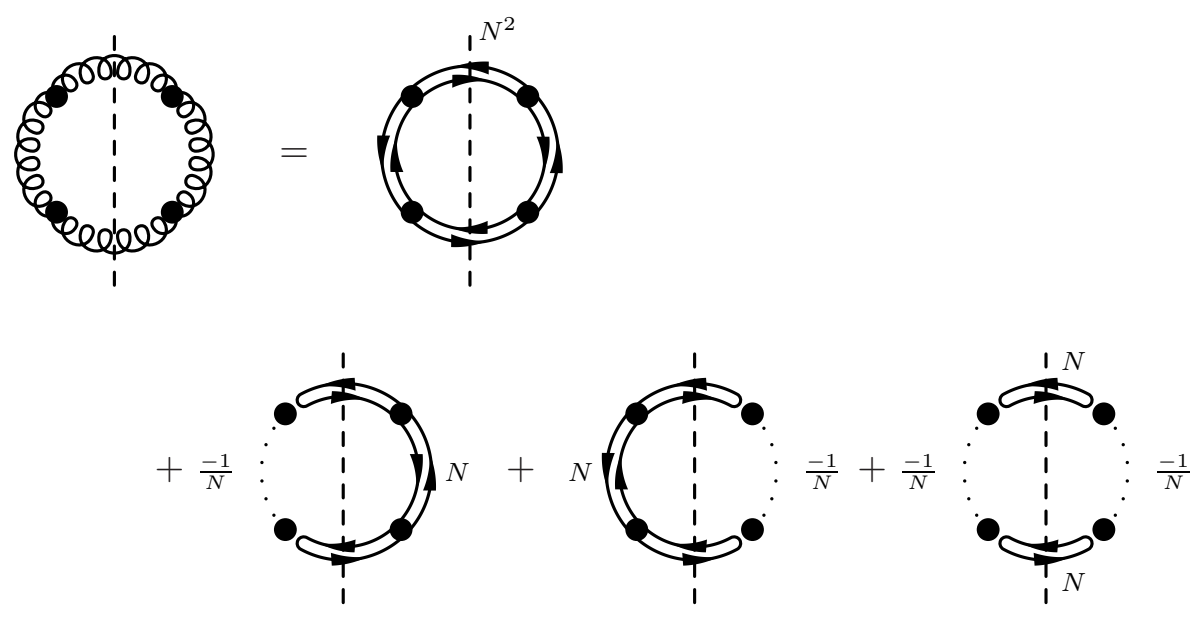

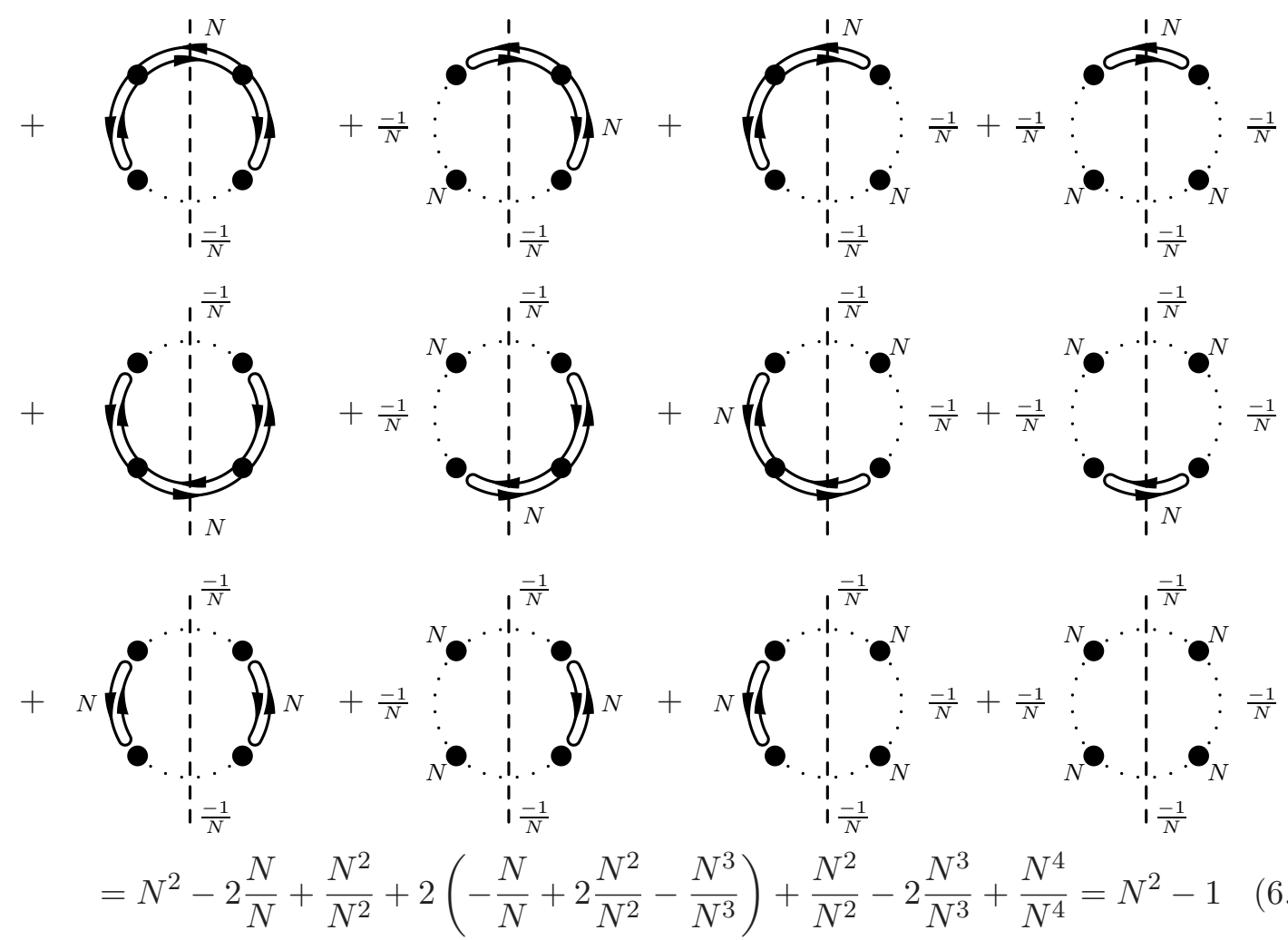

Again, the correct factor is recovered, albeit many cancellations are involved.

At this point, we digress somewhat and discuss a possible way to remove this redundancy before the sum is computed. Depending on the method by which the squared amplitude is computed, this may be useful for improving efficiency.

We would like to apply the direct cancellation between a phantom and a singlet line, where it appears obvious. If the amplitude is completely expanded in terms of diagrams, explicit singlets appear always attached to one of the effective vertices that involve a closed color loop, including the loop pertaining to the effective non-QCD interaction that we are considering here.

Starting at this point (the vertex on the left in the figure), we could apply a procedure that looks like

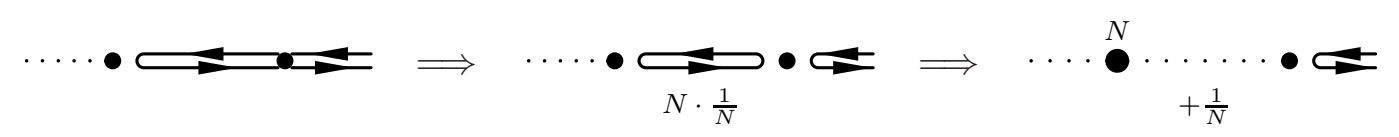

We can replace the color of an isolated singlet gluon that is attached to further gluon lines by a closed color loop, if we divide by $1 / N$. We then remove the closed color line from the diagram, attaching the factor $N$ to the vertex on the left where the singlet originated. The same factor will be present in the analogous diagram where a phantom originates from the vertex. The two contributions now differ only in sign, and thus cancel.

This algorithm is nonlocal in nature and can be implemented, at face value, only if the amplitude is expanded in terms of diagrams. In automatic computational programs that 
construct the amplitude recursively, without expanding Feynman diagrams, the cancellation procedure would have to be implemented as part of the recursive calculation.

In any case, if we apply this argument to the $g g \rightarrow H H$ amplitude before squaring, we obtain the much simpler result

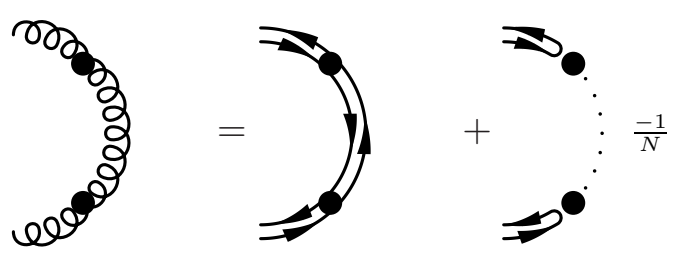

When we compute the square, we have to take into account that singlets, being contained in the $\mathrm{U}(N)$ gluon matrix, interfere with $\mathrm{U}(N)$ gluons. This interference acts as a projection operator which turns a $\mathrm{U}(N)$ gluon into a singlet, on the other side of the cut:
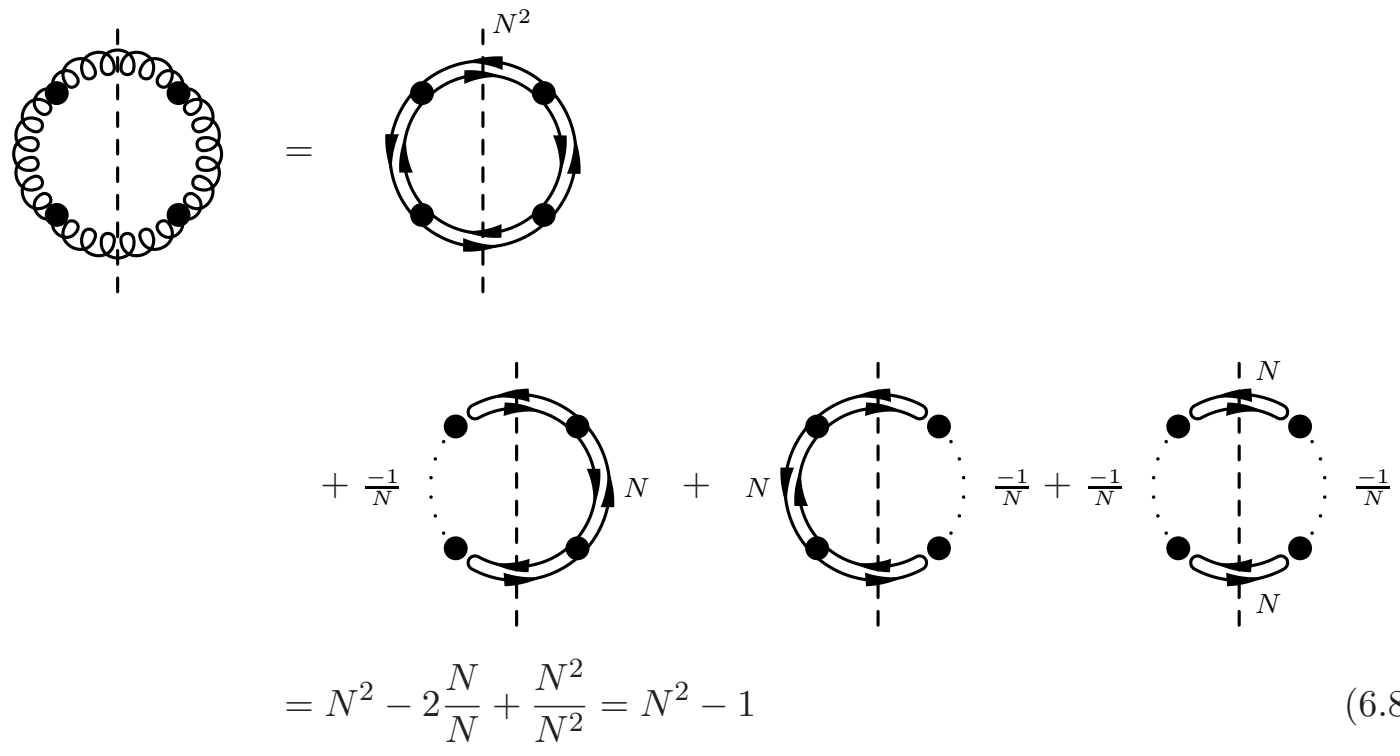

Graphically, we can again apply the cancellation procedure and obtain the final diagrammatic result
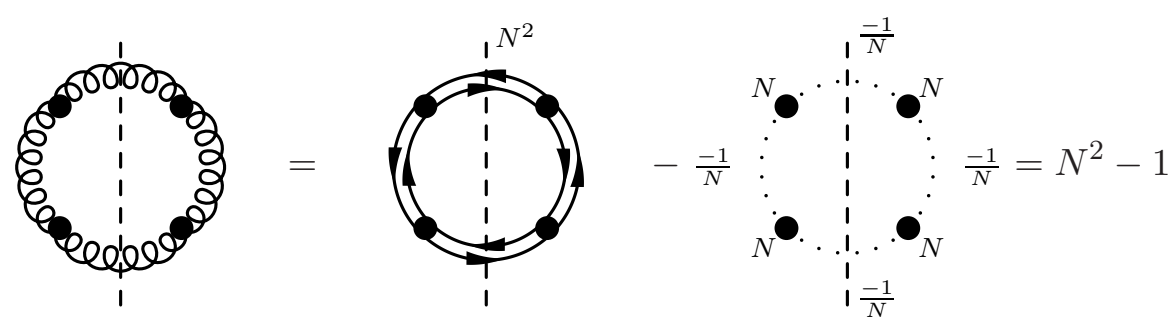

There is no redundancy left. Note that, effectively, the cancellation procedure has switched the sign of the phantom-loop graph, so we would have obtained this result if we just had included this graph with switched sign, but no graphs that explicitly involve singlets. This observation might be generalized and incorporated into the algorithm. 


\section{3 $\quad H \rightarrow g g g$}

The effective one-loop vertex $H \rightarrow g g g$ has two color structures, $f^{a b c}$ and $d^{a b c}$, which originate from the difference and sum of the possible loop orientations, respectively. In the color-flow basis, the Feynman rules are
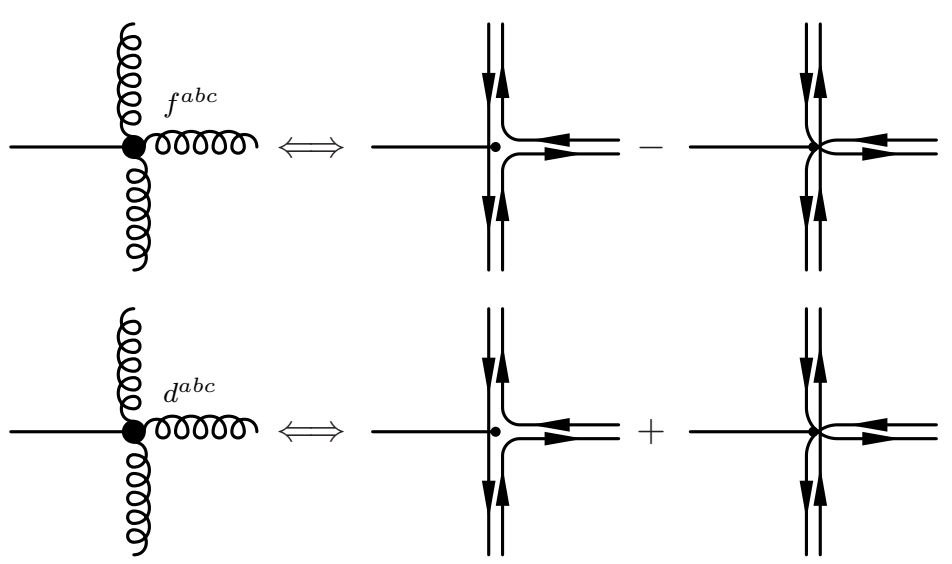

The square of the $f$ term is straightforward:

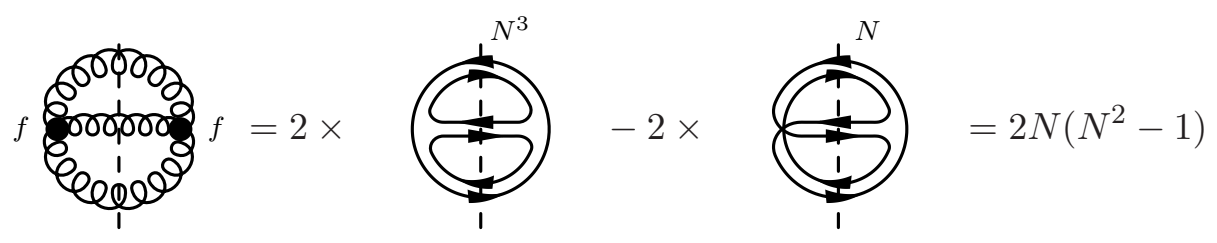

The interference vanishes as expected. The square of the $d$ term yields
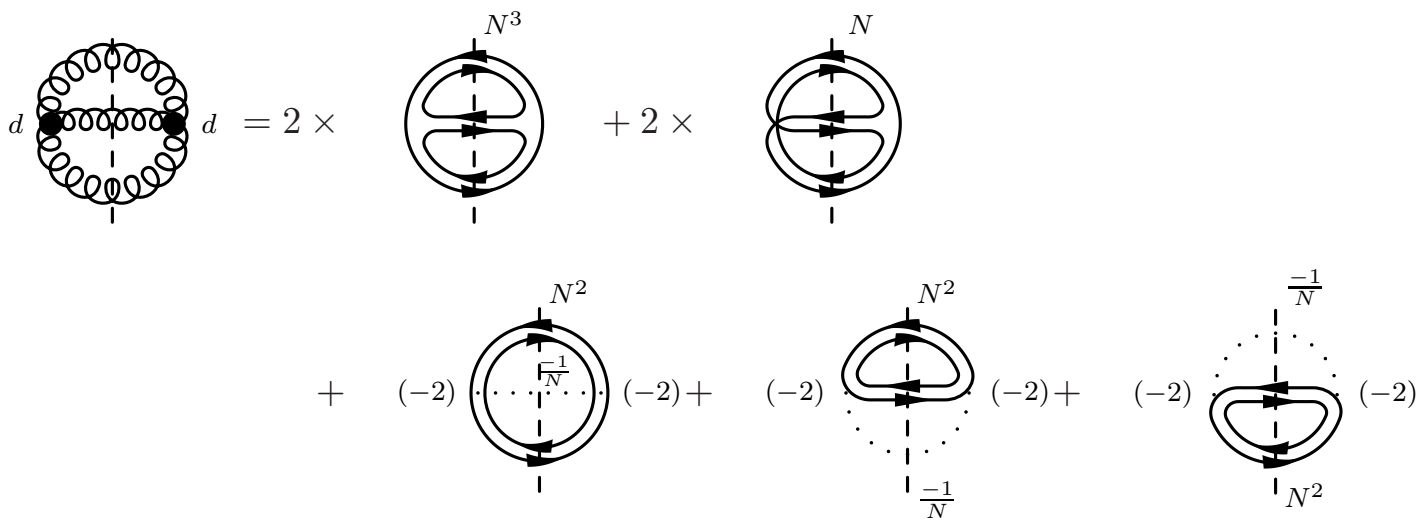


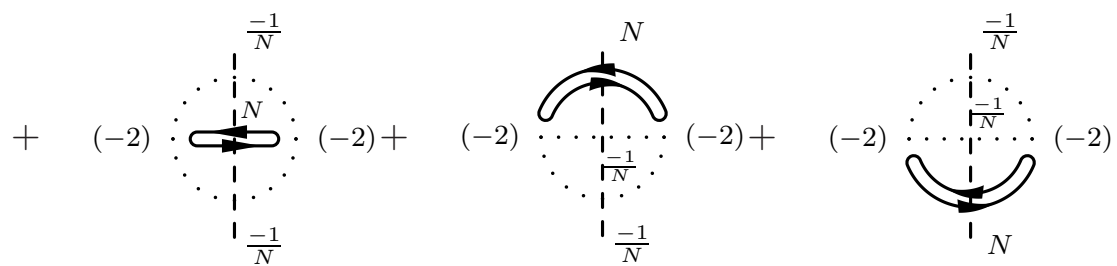

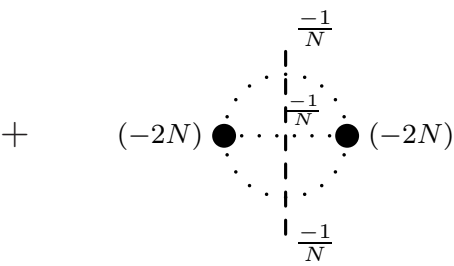

$$
\begin{aligned}
& =2 N^{3}+2 N-12 N+\frac{12}{N}-\frac{4}{N}=2\left(N-\frac{4}{N}\right)\left(N^{2}-1\right)
\end{aligned}
$$

Eliminating the external singlet lines as before would allow us to combine the last two terms, i.e.,

$$
2 N^{3}+2 N-12 N+\frac{8}{N}=2\left(N-\frac{4}{N}\right)\left(N^{2}-1\right)
$$

which is slightly simpler.

\section{Conclusions}

The expansion of QCD amplitudes in a color-flow basis has been known as a useful device in various contexts of perturbative and non-perturbative calculations. In this paper, we have demonstrated that it can also be understood as a field theory of its own, a priori different from, but equivalent to, standard QCD. We have shown that this field theory is well defined, renormalizable, unitary, and yields observable predictions identical to ordinary QCD.

For practical applications, color-flow QCD has advantages in the context of automatic calculations, where it systematically generates color-connected amplitudes that can be matched to parton-shower and hadronization algorithms, avoiding some extra effort that pertains to color algebra and change of bases. In particular, it is useful for algorithms which do not work with a diagrammatic expansion of the amplitude.

We have implemented color-flow QCD in the O'Mega matrix element generator, which is the tool for tree-level amplitude generation contained in the WHIZARD event generator package [22] together with its parton shower generator [45]. The color-flow approach also provides a convenient way of incorporating exotic color interactions. If desired, the methods presented in the present paper can readily be extended to other exotics, e.g., color-decuplet fields.

For higher-order calculations, color-flow QCD can be taken at face value, provided the renormalization procedure is properly implemented (cf. section 4), which is essentially trivial. Concerning mixed QCD-electroweak processes at loop level, the examples in section 6 show that there are minor technical complications, which however do not invalidate the procedure. A comprehensive treatment of NLO calculations would also require a color-flow 
exposition of (dipole) subtraction and parton splitting kernels. This is not discussed in the present paper, but straightforward and part of a different publication [46].

Finally, we remark that the methods developed in the present paper could be generalized and applied to other gauge groups, including exceptional groups, using the completeness relations from [3].

\section{Acknowledgments}

We acknowledge support by the Helmholtz Alliance "Physics at the Terascale". WK has been supported by the BMBF, Contract \#05H09PSE, TO has been supported by the BMBF, Contract \#05H09WWE. CS has been supported by the Deutsche Forschungsgemeinschaft through the Research Training Group GRK 1102 Physics of Hadron Accelerators. WK wants to thank S. Willenbrock and T. Stelzer for illuminating discussions and hospitality at the University of Urbana-Champaign.

\section{A Feynman rules for color-flow QCD}

Here, we list the Feynman rules for $\mathrm{SU}(N)$ gluons interacting with fermions in the fundamental representation, in the color-flow formalism. We shift the coupling $g / \sqrt{2}$ from the kinetic gluon terms in (2.2) and (2.13), respectively, to the vertices by renormalizing gluon and phantom fields accordingly, to obtain canonically normalized kinetic terms. Expanding in terms of components, the color-flow Lagrangian becomes

$$
\begin{aligned}
\mathcal{L}= & -\frac{1}{4}\left(G^{i}{ }_{j}\right)_{\mu \nu}\left(G_{i}^{j}\right)^{\mu \nu}+\frac{N}{4} \tilde{G}_{\mu \nu} \tilde{G}^{\mu \nu}+\bar{\psi}_{i}\left[\mathrm{i} \not \partial \delta_{j}^{i}+\frac{g}{\sqrt{2}}\left(A^{i}{ }_{j}-\tilde{A} \delta_{j}^{i}\right)\right] \psi^{j} \\
& +B^{i}{ }_{j}\left(\partial \cdot A^{j}{ }_{i}\right)-N \tilde{B}(\partial \cdot \tilde{A})+\frac{\xi}{2} B_{j}^{i} B_{i}^{j}-N \frac{\xi}{2} \tilde{B}^{2}+\mathcal{L}_{\text {ghost }}
\end{aligned}
$$

where

$$
\mathcal{L}_{\text {ghost }}=-\bar{c}_{j}^{i} \partial^{\mu}\left(\partial_{\mu} c_{i}^{j}-\frac{g}{\sqrt{2}} \mathrm{i}\left[A_{\mu}, c\right]_{i}^{j}\right)
$$

Note that in the color-flow formalism, the basic coupling emerges as $g / \sqrt{2}$. In the Feynman rules below, Lorentz and momentum factors are omitted, they retain their usual form. Propagators:

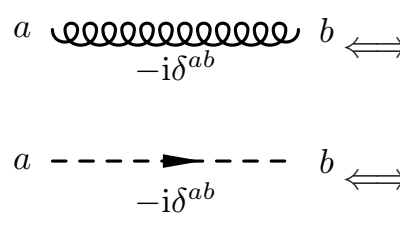

$$
\begin{aligned}
& { }_{i}^{j} \underset{-\mathrm{i} \delta_{k}^{i} \delta_{j}^{l}}{{ }_{k}^{l}} \quad \begin{array}{c}
\ldots \\
-\mathrm{i}\left(\frac{-1}{N}\right)
\end{array} \\
& { }_{i}^{j} \quad=-\frac{--}{-\mathrm{i} \delta_{k}^{i} \delta_{j}^{l}}== \pm{ }_{k}^{l}
\end{aligned}
$$


Vertices:
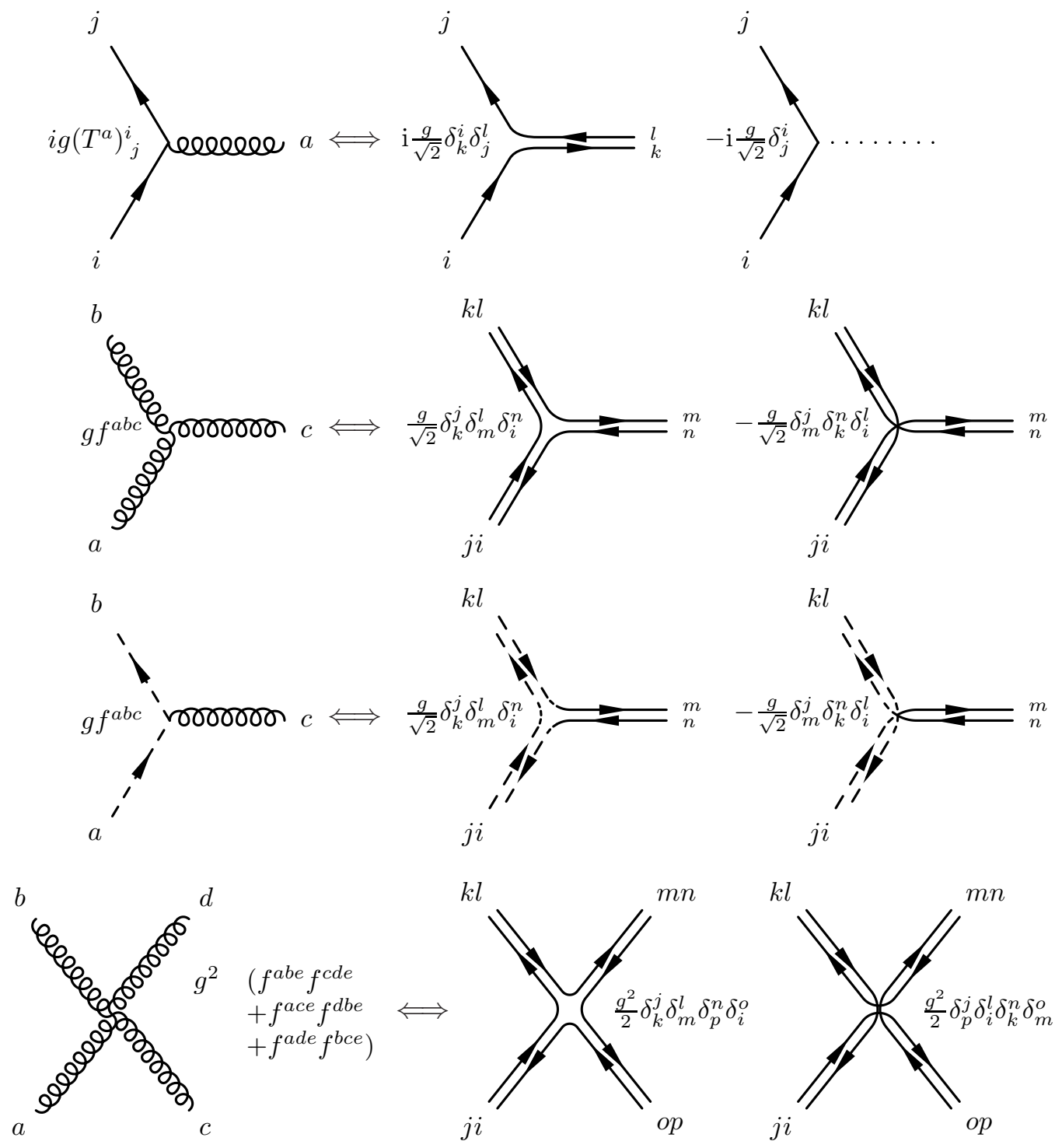

(A.8)
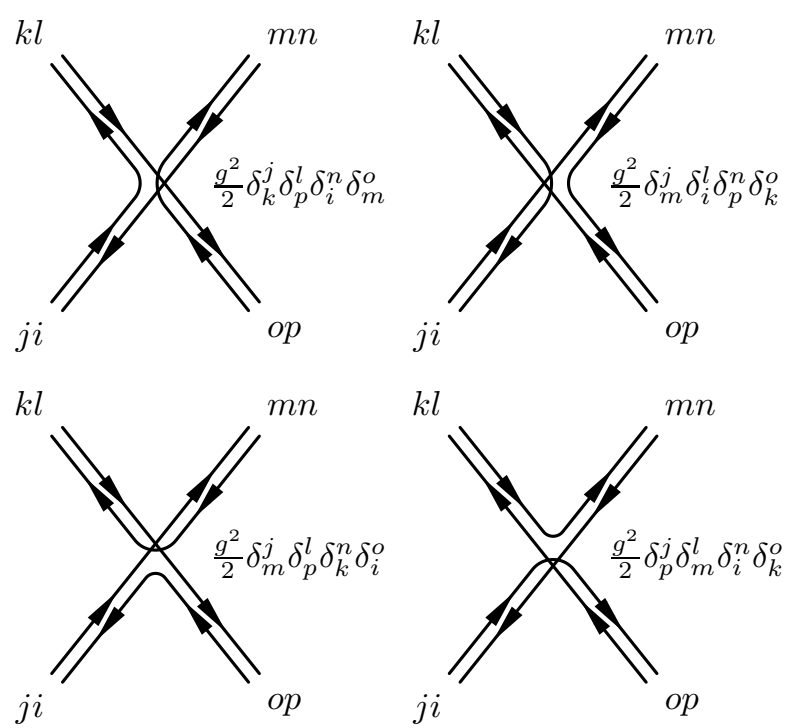


\section{B Color-sextet particles}

QCD and the Standard Model contain colored fields only in the fundamental and in the adjoint representation of SU(3). However, various Standard Model extensions that are currently under discussion provide extra colored fields and interactions. Automatic computation programs should be able to deal with such extensions, and the color-flow approach provides a straightforward means to achieve this. As a particular example, we describe the color-flow Feynman rules for color-sextet scalar particles.

Color-sextet particles or, more generally, particles in the symmetric bi-fundamental representation of $\mathrm{SU}(N)$ might exist at mass scales accessible at the LHC. A possible source are extended Higgs (super-)multiplets in unified gauge theories of the strong and electroweak interactions.

We consider a color-sextet scalar particle denoted as $\sigma$. An explicit representation is a complex symmetric matrix in color space with six independent entries $\sigma_{1,2,3}$ and $\bar{\sigma}_{1,2,3}$,

$$
\sigma=\left(\begin{array}{ccc}
\sigma_{1} & \frac{1}{\sqrt{2}} \bar{\sigma}_{3} & \frac{1}{\sqrt{2}} \bar{\sigma}_{2} \\
\frac{1}{\sqrt{2}} \bar{\sigma}_{3} & \sigma_{2} & \frac{1}{\sqrt{2}} \bar{\sigma}_{1} \\
\frac{1}{\sqrt{2}} \bar{\sigma}_{2} & \frac{1}{\sqrt{2}} \bar{\sigma}_{1} & \sigma_{3}
\end{array}\right)
$$

with components $\sigma_{i j}$, where $\sigma_{i j}=\sigma_{j i}$. We also need the antiparticle $\sigma^{i j}$. This particle couples to gluons and gluon pairs. It may also couple to colorless states (e.g., Higgs), and linearly to quark pairs. The Lagrangian is

$$
\mathcal{L}=D_{\mu} \sigma_{i j} D^{\mu} \sigma^{i j}-m^{2} \sigma_{i j} \sigma^{i j}-\lambda H \sigma_{i j} \sigma^{i j}-g_{q q^{\prime}}\left(\sigma^{i j} \bar{q}_{i}\left(q_{j}^{\prime}\right)^{c}+\text { h.c. }\right)
$$

The covariant derivative, in color-flow QCD, is given by

$$
D_{\mu} \sigma=\partial_{\mu} \sigma-\mathrm{i}\left(A_{\mu} \sigma+\sigma A_{\mu}^{T}-2 \tilde{A}_{\mu} \sigma\right)
$$

We derive the Feynman rules. The propagator has two components which symmetrize the color flow:

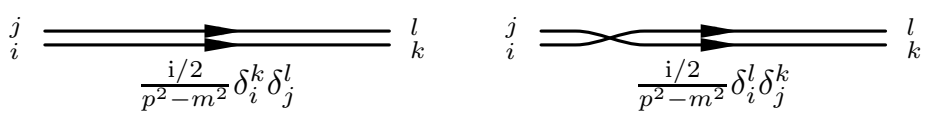

The same symmetrization is needed for external sextet particles.

Gluons interact symmetrically with both color lines. The single-gluon interaction splits into three distinct Feynman rules:
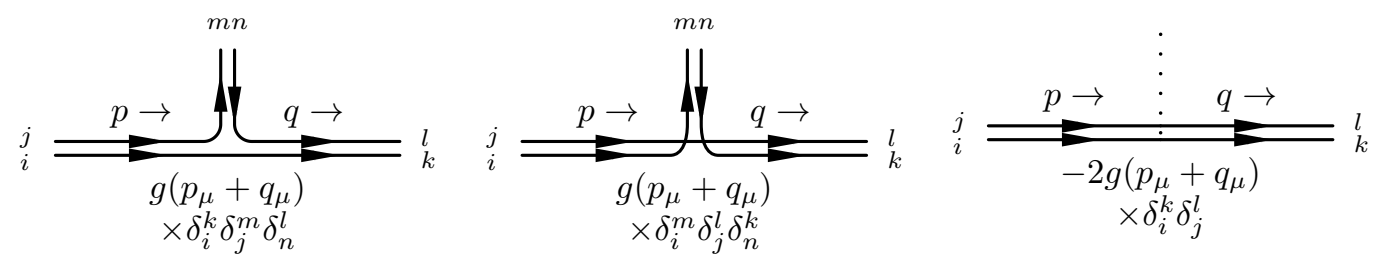
The double-gluon interaction splits into six distinct Feynman rules:
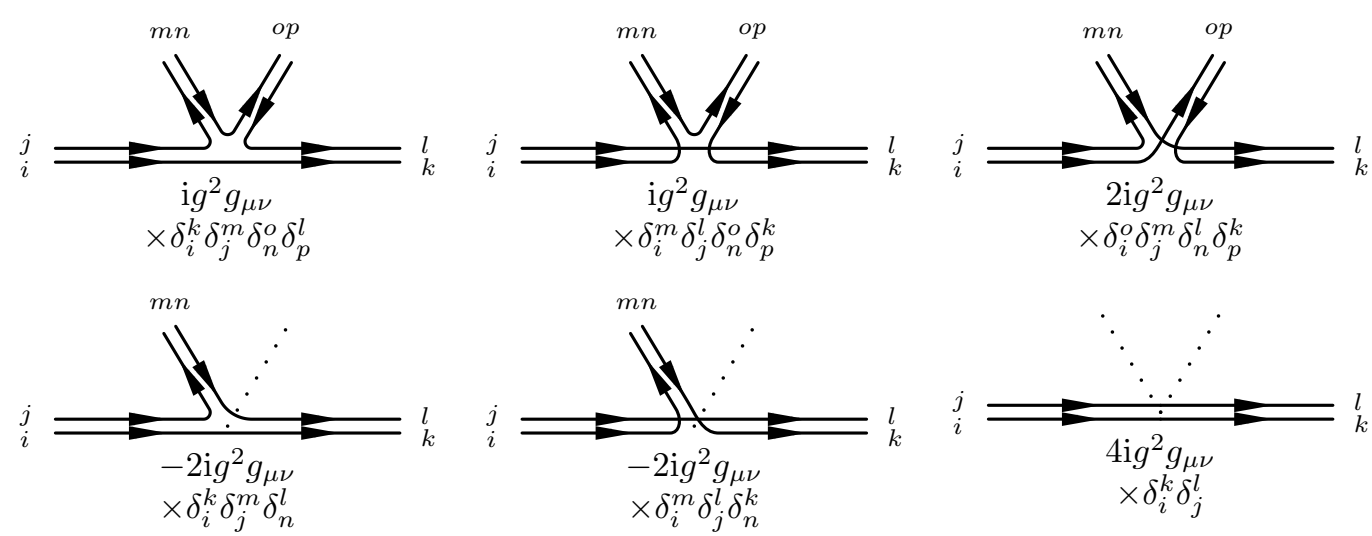

Couplings to colorless particles are simply

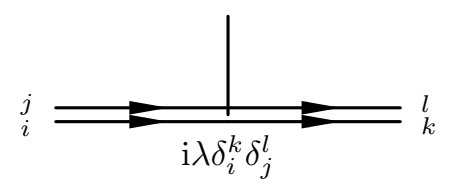

and the coupling to quark pairs has the Feynman rules
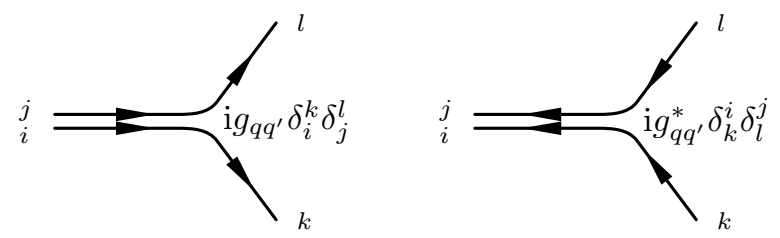

Open Access. This article is distributed under the terms of the Creative Commons Attribution License which permits any use, distribution and reproduction in any medium, provided the original author(s) and source are credited.

\section{References}

[1] G. 't Hooft, A Planar Diagram Theory for Strong Interactions, Nucl. Phys. B 72 (1974) 461 [INSPIRE].

[2] G. Canning, Color Symmetry in the Large-N Limit, Phys. Rev. D 12 (1975) 2505 [INSPIRE].

[3] P. Cvitanovic, Group Theory for Feynman Diagrams in Nonabelian Gauge Theories: Exceptional Groups, Phys. Rev. D 14 (1976) 1536 [INSPIRE].

[4] R. Penrose, Applications of negative dimensional tensors, in Combinatorial Mathematics and its Applications (Proc. Conf., Oxford 1969), D.J.A. Welsh ed., Academic Press, New York U.S.A. (1971).

[5] P. Cvitanovic, Group theory: Birdtracks, Lie's and exceptional groups, Princeton University Press, Princeton U.S.A. (2008).

[6] H. Harari, Duality Diagrams, Phys. Rev. Lett. 22 (1969) 562 [inSPIRE].

[7] J.L. Rosner, Graphical Form of Duality, Phys. Rev. Lett. 22 (1969) 689 [INSPIRE]. 
[8] B. Andersson and G. Gustafson, Semiclassical Models for Gluon Jets and Leptoproduction Based on the Massless Relativistic String, Z. Phys. C 3 (1980) 223 [InSPIRE].

[9] G. Gustafson, Topological properties of the color field in hard processes, Z. Phys. C 15 (1982) 155 [inSPIRE].

[10] H. Bengtsson, The Lund Monte Carlo For High $p_{T}$ Physics, Comput. Phys. Commun. 31 (1984) 323 [INSPIRE].

[11] R.K. Ellis, G. Marchesini and B. Webber, Soft Radiation in Parton Parton Scattering, Nucl. Phys. B 286 (1987) 643 [Erratum ibid. B 294 (1987) 1180] [INSPIRE].

[12] G. Marchesini and B. Webber, Monte Carlo Simulation of General Hard Processes with Coherent QCD Radiation, Nucl. Phys. B 310 (1988) 461 [InSPIRE].

[13] T. Sjöstrand et al., QCD Generators, in Z Physics at LEP 1, G. Altarelli, R. Kleiss and C. Verzegnassi eds., CERN 89-08, Geneva (1989), Vol. 3 pg. 143.

[14] J. Bell and R. Jackiw, A PCAC puzzle: $\pi^{0} \rightarrow \gamma \gamma$ in the $\sigma$-model, Nuovo Cim. A 60 (1969) 47 [inSPIRE].

[15] S.L. Adler, Axial vector vertex in spinor electrodynamics, Phys. Rev. 177 (1969) 2426 [INSPIRE].

[16] E. Boos et al., Generic user process interface for event generators, hep-ph/0109068 [INSPIRE].

[17] J. Alwall et al., A Standard format for Les Houches event files, Comput. Phys. Commun. 176 (2007) 300 [hep-ph/0609017] [INSPIRE].

[18] F. Maltoni, K. Paul, T. Stelzer and S. Willenbrock, Color flow decomposition of QCD amplitudes, Phys. Rev. D 67 (2003) 014026 [hep-ph/0209271] [InSPIRE].

[19] F. Caravaglios and M. Moretti, An algorithm to compute Born scattering amplitudes without Feynman graphs, Phys. Lett. B 358 (1995) 332 [hep-ph/9507237] [INSPIRE].

[20] A. Kanaki and C.G. Papadopoulos, HELAC: A Package to compute electroweak helicity amplitudes, Comput. Phys. Commun. 132 (2000) 306 [hep-ph/0002082] [INSPIRE].

[21] M. Moretti, T. Ohl and J. Reuter, O'Mega: An Optimizing matrix element generator, in 2nd ECFA/DESY Study 1998-2001, [hep-ph/0102195] [INSPIRE].

[22] W. Kilian, T. Ohl and J. Reuter, WHIZARD: Simulating Multi-Particle Processes at LHC and ILC, Eur. Phys. J. C 71 (2011) 1742 [arXiv:0708.4233] [InSPIRE].

[23] A. Cafarella, C.G. Papadopoulos and M. Worek, Helac-Phegas: A Generator for all parton level processes, Comput. Phys. Commun. 180 (2009) 1941 [arXiv:0710.2427] [INSPIRE].

[24] K. Hagiwara and Y. Takaesu, Generating QCD amplitudes in the color-flow basis with MadGraph, Eur. Phys. J. C 71 (2011) 1668 [arXiv:1010.0748] [INSPIRE].

[25] L. Faddeev and V. Popov, Feynman Diagrams for the Yang-Mills Field, Phys. Lett. B 25 (1967) 29 [INSPIRE].

[26] N. Nakanishi, Covariant quantization of the electromagnetic field in the Landau gauge, Prog. Theor. Phys. 35 (1966) 1111 [InSPIRE].

[27] B. Lautrup, Canonical Quantum Electrodynamics in Covariant Gauges, K. Dan. Vidensk. Selsk. Mat.-Fys. Medd. 35 (1967) 11. 
[28] C. Becchi, A. Rouet and R. Stora, The Abelian Higgs-Kibble Model. Unitarity of the S Operator, Phys. Lett. B 52 (1974) 344 [INSPIRE].

[29] C. Becchi, A. Rouet and R. Stora, Renormalization of the Abelian Higgs-Kibble Model, Commun. Math. Phys. 42 (1975) 127 [InSPIRE].

[30] C. Becchi, A. Rouet and R. Stora, Renormalization of Gauge Theories, Annals Phys. 98 (1976) 287 [INSPIRE].

[31] I. Tyutin, Gauge Invariance in Field Theory and Statistical Physics in Operator Formalism, arXiv: 0812.0580 [INSPIRE].

[32] T. Kugo and I. Ojima, Manifestly Covariant Canonical Formulation of Yang-Mills Field Theories: Physical State Subsidiary Conditions and Physical S Matrix Unitarity, Phys. Lett. B 73 (1978) 459 [inSPIRE].

[33] T. Kugo and I. Ojima, Local Covariant Operator Formalism of Nonabelian Gauge Theories and Quark Confinement Problem, Prog. Theor. Phys. Suppl. 66 (1979) 1 [InSPIRE].

[34] T. Kugo, Gauge ba no ryōshiron, Baifukan, Japan (1989), German translation: Eichtheorie, Springer, (1997).

[35] O. Piguet and A. Rouet, Symmetries in Perturbative Quantum Field Theory, Phys. Rept. 76 (1981) 1 [INSPIRE].

[36] O. Piguet and S.P. Sorella, Algebraic renormalization: Perturbative renormalization, symmetries and anomalies, Lect. Notes Phys. M 28 (1995) 1.

[37] J. Lowenstein, Differential vertex operations in Lagrangian field theory, Commun. Math. Phys. 24 (1971) 1 [INSPIRE].

[38] Y.-M.P. Lam, Perturbation Lagrangian theory for scalar fields: Ward-Takahasi identity and current algebra, Phys. Rev. D 6 (1972) 2145 [INSPIRE].

[39] Y.-M.P. Lam, Equivalence theorem on Bogolyubov-Parasiuk-Hepp-Zimmermann renormalized Lagrangian field theories, Phys. Rev. D 7 (1973) 2943 [INSPIRE].

[40] T.E. Clark and J.H. Lowenstein, Generalization of Zimmermann's Normal-Product Identity, Nucl. Phys. B 113 (1976) 109 [InSPIRE].

[41] P. Breitenlohner and D. Maison, Dimensional Renormalization and the Action Principle, Commun. Math. Phys. 52 (1977) 11 [inSPIRE].

[42] P. Breitenlohner and D. Maison, Dimensionally Renormalized Green's Functions for Theories with Massless Particles. 1., Commun. Math. Phys. 52 (1977) 39 [InSPIRE].

[43] P. Breitenlohner and D. Maison, Dimensionally Renormalized Green's Functions for Theories with Massless Particles. 2., Commun. Math. Phys. 52 (1977) 55 [InSPIRE].

[44] E. Witten, Baryons in the 1/n Expansion, Nucl. Phys. B 160 (1979) 57 [INSPIRE].

[45] W. Kilian, J. Reuter, S. Schmidt and D. Wiesler, An Analytic Initial-State Parton Shower, JHEP 04 (2012) 013 [arXiv:1112.1039] [INSPIRE].

[46] J. Reuter and C. Speckner, Dipole subtraction terms in the colorflow basis in WHIZARD, in preparation. 